\title{
Joint analysis of ESR lineshapes and ${ }^{1} \mathrm{H}$ NMRD profiles of DOTA-Gd derivatives by means of the slow motion theory
}

\author{
D. Kruk, ${ }^{1}$,a) J. Kowalewski, ${ }^{2}$ D. S. Tipikin, ${ }^{3}$ J. H. Freed, ${ }^{3}$ M. Mościcki, ${ }^{1,3}$ A. Mielczarek, ${ }^{1,4}$ \\ and M. Port ${ }^{5}$ \\ ${ }^{1}$ Institute of Physics, Jagiellonian University, Reymonta 4, PL-30-059 Krakow, Poland \\ ${ }^{2}$ Department of Materials and Environmental Chemistry, Arrhenius Laboratory, Stockholm University, \\ S-106 91 Stockholm, Sweden \\ ${ }^{3}$ Department of Chemistry and Chemical Biology, Cornell University, Ithaca, New York 14853-1301, USA \\ ${ }^{4}$ Institute of Nuclear Physics, PAS, Radzikowskiego 152, PL-31-342 Krakow, Poland \\ ${ }^{5}$ Guerbet, Research Department, F-95943 Roissy Cdg Cedex, France
}

(Received 18 August 2010; accepted 26 October 2010; published online 11 January 2011; corrected 9 February 2011)

\begin{abstract}
The "Swedish slow motion theory" [Nilsson and Kowalewski, J. Magn. Reson. 146, 345 (2000)] applied so far to Nuclear Magnetic Relaxation Dispersion (NMRD) profiles for solutions of transition metal ion complexes has been extended to ESR spectral analysis, including in addition $g$-tensor anisotropy effects. The extended theory has been applied to interpret in a consistent way (within one set of parameters) NMRD profiles and ESR spectra at 95 and $237 \mathrm{GHz}$ for two Gd(III) complexes denoted as P760 and P792 (hydrophilic derivatives of DOTA-Gd, with molecular masses of 5.6 and $6.5 \mathrm{kDa}$, respectively). The goal is to verify the applicability of the commonly used pseudorotational model of the transient zero field splitting (ZFS). According to this model the transient ZFS is described by a tensor of a constant amplitude, defined in its own principal axes system, which changes its orientation with respect to the laboratory frame according to the isotropic diffusion equation with a characteristic time constant (correlation time) reflecting the time scale of the distortional motion. This unified interpretation of the ESR and NMRD leads to reasonable agreement with the experimental data, indicating that the pseudorotational model indeed captures the essential features of the electron spin dynamics. (C) 2011 American Institute of Physics. [doi:10.1063/1.3516590]
\end{abstract}

\section{INTRODUCTION}

Nuclear magnetic resonance (NMR) paramagnetic relaxation enhancement (PRE) refers to an enhancement of spinlattice relaxation of solvent nuclei (typically water protons) due to the presence of paramagnetic species (transition metal complexes) in solution. The nuclear (proton) spin relaxation is enhanced due to a strong electron-proton dipole-dipole coupling, modulated by the complex reorientation as well as electron spin dynamics. Therefore, the field dependence of the PRE (commonly referred to as Nuclear Magnetic Relaxation Dispersion, NMRD, profile) carries potentially a wealth of information on structure and dynamics of the species involved. In order to extract such information from the experimental data, an appropriate theoretical treatment is needed. The difficult part of such a treatment is to describe the electron spin relaxation processes. A simple approach to the electron spin relaxation and the PRE was proposed long time ago by Bloembergen and Morgan. ${ }^{1}$ Their model was derived for simple hydrated transition metal ions with electron spin quantum number of unity or larger and involved an assumption that the electron spin relaxation was caused by solventinduced fluctuations of the complex geometry. The geometry variation led to a rapidly oscillating (transient) zero field

\footnotetext{
a) Author to whom correspondence should be addressed. Electronic mail: danuta.kruk@uj.edu.pl.
}

splitting (ZFS), averaged to zero on the time scale of molecular rotations, which resulted in the electron spin relaxation as simple exponential processes. This approach became, in the context of PRE, the essential part of the well-known modified Solomon-Bloembergen equations and the SolomonBloembergen-Morgan (SBM) theory. ${ }^{2-4}$ Even then, the assumption of a single exponential electron spin relaxation in high-spin systems causes some problems outside of the extreme narrowing regime. ${ }^{5}$ The multiexponential electron spin relaxation and its effect on the PRE were considered by Rubinstein et al. ${ }^{6}$ and Westlund and Strandberg. ${ }^{7,8}$ Further theoretical efforts developed into two directions. The first kind of theoretical treatment of the PRE is based on the perturbation theory for the electron spin system and is therefore subject to serious limitations. The underlying assumption of the perturbation approaches is that the electron spin subsystem fulfils the conditions of Redfield relaxation theory ${ }^{5,9,10}$ which considerably narrows their validity regimes. An important example of the perturbation treatments is the inner-sphere PRE theory (the inner-sphere contribution comes from the solvent molecules entering the first coordination shell of the metal ion) for slowly rotating systems of the electron spin quantum number $S=1,{ }^{11}$ which was later generalized to an arbitrary spin quantum number also including the outer-sphere PRE (the contribution of the molecules outside of this shell). ${ }^{12}$ Another perturbation approach was presented by Sharp et al., ${ }^{13-18}$ accounting for low-field features of the electron 
spin relaxation. Rast et al. proposed a theoretical description of the electron spin relaxation, still based on the Redfield relaxation theory, including the effects of a permanent (static) ZFS ${ }^{19,20}$ and allowing also for higher order terms. That model was applied for interpretation of NMRD profiles and electron spin resonance (ESR) lineshapes in small Gd(III) complexes.

The perturbation approaches give a valuable insight into the electron spin relaxation and its effect on the nuclear spin relaxation, but many systems violate the assumptions of the perturbation theory. Amplitudes of the ZFS interactions combined with motional conditions bring the electron spin beyond validity regimes of the Redfield theory and the electron spin relaxation times cannot explicitly be defined. Rast et al. ${ }^{21}$ proposed one way to resolve the electron dynamics problem and Borel et al. ${ }^{22}$ applied it to unified analysis of ESR and NMRD data for some small Gd(III) complexes.

A general category of (slow motion) treatments is based on the stochastic Liouville equation (SLE). The name "slow motion" originates from the situation when the mean interaction strength is larger than, or comparable to, the inverse of the correlation time that corresponds to the motion modulating the interaction. The methods based on the stochastic Liouville equation were introduced by Freed and co-workers ${ }^{23-28}$ to describe ESR lineshapes for systems with the electron spin quantum number $S=1 / 2$, including interactions with neighboring nuclear spins under very general anisotropic motional conditions. A related approach was also applied to describe inner-sphere NMRD for transition metal complexes, starting from the case of $S=1$ (Ref. 29) and later generalized to an arbitrary spin quantum number. ${ }^{30}$ This treatment is referred to in the literature as "the Swedish slow motion theory". In this context, one should mention somewhat different implementations of the slow motion theory, SLE-L, (stochastic Liouville equation in Langevin form) presented by Åman and Westlund. ${ }^{31,32}$ Predictions of the slow motion theory ${ }^{30}$ were recently compared ${ }^{33}$ with two other treatments, referred to as the Grenoble, ${ }^{21,34}$ and Ann Arbor approaches, ${ }^{35-37}$ respectively. It was found that the "Swedish slow motion theory" and the Grenoble approach agreed very well with each other, while some discrepancies were observed when compared to the Ann Arbor method, which was explained by a somewhat different description of the electron spin dynamics. Recently, this approach was extended to the outer-sphere $\mathrm{PRE}^{38}$ (employing the model of translational diffusion proposed a long time ago by Hwang and Freed ${ }^{39}$ and Ayant et al. ${ }^{40}$ ), resulting in a complete tool for analyzing NMRD profiles for $S \geq 1$ for isotropic reorientation over a broad range of rates. The SLE-based formalism was early applied to calculate ESR lineshapes for $S=1$ over the whole motional range. ${ }^{41}$ More recently, it was used to study electron spin relaxation for $S=1$ at low field. ${ }^{42}$ Here, we generalize the approach to high spin systems at arbitrary magnetic fields.

The three NMRD models compared above ${ }^{33}$ are based on the same description of the ZFS interactions. The ZFS coupling is split into a permanent (static) part modulated by the molecular tumbling and a fluctuating (transient) part varying in time mostly by the distortional (vibrational) motion of the complex. The transient ZFS is modeled as a tensor of a constant amplitude, defined in its own principal axis sys- tem, which changes its orientation with respect to a regular molecule-fixed frame according to the isotropic diffusion equation with a characteristic time constant (correlation time) reflecting the time scale of the distortional motion. ${ }^{3,4,6,30}$ The modulation of the transient ZFS interaction is the principal origin of the electron spin dynamics. At the same time, the pseudorotational model is an obvious oversimplification, which was amply demonstrated by molecular dynamics (MD) simulations. $^{43-45}$ More complex descriptions of the distortional motion and, in consequence, the fluctuating part of the ZFS, were proposed ${ }^{46,47}$ and incorporated into the slow motion theory (so far for the electron spin quantum number $S=1$ ). These models are based on classical and quantummechanical description of the distortional (vibrational) motion in terms of normal modes. Nevertheless, even though one is willing to take a considerable computational effort to describe more realistically the electron spin dynamics, the number of parameters needed for that leads to serious limitations of such approaches. Such an analysis has to be supported by, for example, molecular dynamics calculations in order to provide an independent estimation of the relevant parameters. ${ }^{43,44}$ The pseudorotational model is commonly used because of its relatively simple mathematical formulation and because it only requires the amplitudes of the transient ZFS (in principle its axial and rhombic parts, but the last one is usually neglected) and the characteristic correlation time. In this context, the question whether the pseudorotational model captures the essential features of the electron spin dynamics becomes very important. A way to verify this point is to attempt a unified interpretation of multifrequency ESR spectra and NMRD profiles within one set of parameters. Such attempts have been undertaken in the past. Rubinstein et $a l .{ }^{6}$ used their model to calculate ESR linewidths and PRE for $\mathrm{Cr}(\mathrm{III}), \mathrm{Fe}(\mathrm{III})$, and $\mathrm{Mn}$ (II) in water solution. Powell et $a l .{ }^{48}$ were the first to apply the SBM theory to the combined data sets for a number of Gd(III) complexes, with moderate success. Similar approaches were also adopted for other Gd(III) chelates by Toth et al. ${ }^{49}$ and, more recently ZithaBovens et al. ${ }^{50}$ The improved perturbation theory for electron spin relaxation ${ }^{19,20}$ turned out to be more successful for the aqueous $\mathrm{Gd}(\mathrm{III})$ ion $^{21,22,51,52}$ and other small $\mathrm{Gd}(\mathrm{III})$ complexes. ${ }^{53}$ Larger complexes, with electron spin relaxation outside of the Redfield limit, can be expected to be more difficult. Zhou and co-workers described ESR spectra and NMRD data for the Gd(III) complex MS-325+HAS, applying the modified SBM approach. ${ }^{54,55}$ This attempt did not turn out as very successful, because (as the authors pointed out in their conclusions ${ }^{54}$ ) the analyzed system was in the slow motion regime, while the modified SBM approach is based on the perturbation theory. Even though detailed ESR experimental studies allow for an independent determination of the ZFS parameters, including their sign, ${ }^{56}$ the question of the applicability of the pseudorotational model as a reliable way to describe the electron spin dynamics still remains open. From a different starting point, a very interesting model of the transient ZFS affected by an Ornstein-Uhlenbeck process with a Gaussian probability distribution has been proposed for ESR spectral analysis ${ }^{57}$ but (at least so far) not tested against NMRD data. 
In this work, we attempt to critically verify the applicability of the pseudorotational model for a system in the slow motion regime. First we extend the "Swedish slow motion theory" to ESR spectral analysis contributing in this way to the general theoretical tool for jointly interpreting ESR and NMRD data for highly variable motional conditions. Then we apply the extended theory to interpret multifrequency ESR spectra and NMRD data of some derivatives of DOTA-Gd.

The outline of this paper is as follows. In Sec. II A, we review some basic concepts of the PRE theory in general, while in Secs. II B and II C we describe its extension to ESR spectral analysis. This is followed by the attempt of a unified analysis of ESR and NMRD data (Sec. III) and discussion (Sec. IV), conclusions are drawn in Sec. V.

\section{II. "SLOW MOTION THEORY” FOR NMRD AND ESR}

The "Swedish slow motion theory" has been so far applied to interpret NMRD profiles for various paramagnetic systems. Here we have adapted this approach to ESR lineshape analysis. The principles of the slow motion theory have been explained in great detail in several papers, for example Refs. 3, 4, and 25, and 30. Nevertheless, we sum up here the main steps of the slow motion treatment, before we proceed with the ESR description.

\section{A. Recapitulation of the slow motion approach to NMRD}

As it has already been explained in the Introduction, the underlying assumption of the "slow motion theory" of the paramagnetic relaxation enhancement effect is that the nuclear spin subsystem fulfills conditions of a perturbation approach to relaxation. In fact, this assumption is pretty obvious; otherwise one could not define the nuclear spin relaxation times/rates. More exactly, it is assumed that the nuclear spin fulfills the Redfield condition ${ }^{5,9,10}$ that implies that the Redfield relaxation theory can be applied to the nuclear spin relaxation. In consequence the nuclear (proton) spin lattice relaxation rate, resulting from the PRE effect, $R_{1 I}^{\mathrm{PRE}}\left(\omega_{I}\right)(I$ denotes the nuclear spin) at the proton Larmor frequency $\omega_{I}$, caused by a dipole-dipole coupling between the nuclear and electron spins, is given as: $R_{1 I}^{\mathrm{PRE}}\left(\omega_{I}\right)=2 \operatorname{Re}\left\{K_{1,1}^{D D}\left(-\omega_{I}\right)\right\}$. $3,4,29,30$ The lattice spectral density function $K_{1,1}^{D D}\left(-\omega_{I}\right)$ is defined as: $3,4,29,30$

$$
\begin{aligned}
K_{1,1}^{D D}\left(-\omega_{I}\right)= & \int_{0}^{\infty} \operatorname{Tr}_{L}\left\{T_{1}^{1(D D)+}\left[\exp \left(-i \hat{\hat{L}}_{\mathrm{L}} \tau\right) T_{1}^{1(D D)}\right] \rho_{\mathrm{L}}^{\mathrm{eq}}\right\} \\
& \times \exp \left(-i \omega_{I} \tau\right) d \tau .
\end{aligned}
$$

This formula requires some explanation. In the high temperature approximation the equilibrium density matrix operator $\rho_{\mathrm{L}}^{\text {eq }}$ can be set to $\rho_{\mathrm{L}}^{\text {eq }}=1 /(2 S+1)$. The lattice Liouville operator, $\hat{\hat{L}}_{\mathrm{L}}$, contains all degrees of freedom which are relevant for the nuclear spin relaxation. In the framework of the slow motion theory of the inner-sphere PRE this operator includes the following terms: $:^{3,4,30} \hat{\hat{L}}_{\mathrm{L}}=\hat{\hat{L}}_{\mathrm{Z}}(S)$ $+\hat{\hat{L}}_{\mathrm{ZFS}}^{S}+\hat{\hat{L}}_{\mathrm{ZFS}}^{T}+\hat{\hat{L}}_{D}+\hat{\hat{L}}_{R}$. The contributing operators rep- resent the Zeeman interaction for the electron spin $(S)$, the static and transient zero field splitting, distortional and rotational motions of the complex. The Zeeman Hamiltonian, $H_{Z}(S)$ associated with the Liouville operator, $\hat{\hat{L}}_{Z}(S)$, has the obvious form: $H_{Z}(S)=\omega_{S} S_{Z}\left(\omega_{S}\right.$ is the electron Larmor frequency). The forms of the static and transient ZFS (in the laboratory frame) depend on the models of motion incorporated into the theory. The static (permanent) zero field splitting is a part of the entire ZFS interaction, $H_{\mathrm{ZFS}}(t)=$ $H_{\mathrm{ZFS}}^{S}(t)+H_{\mathrm{ZFS}}^{T}(t)$, obtained as a result of averaging over complex distortions (vibrations). According to the models incorporated into the slow motion theory, it is represented in the laboratory frame by the Hamiltonian:

$$
H_{\mathrm{ZFS}}^{S(L)}(t)=\sum_{m=-2}^{2}(-1)^{m} V_{-m}^{2 S(L)}(t) T_{m}^{2}(S)
$$

with

$$
\begin{aligned}
V_{-m}^{2 S(L)}(t)= & \sqrt{\frac{2}{3}} D_{S} D_{0,-m}^{2}\left(\Omega_{P_{S} L}(t)\right)+E_{S}\left[D_{-2,-m}^{2}\left(\Omega_{P_{S} L}\right)(t)\right. \\
& \left.+D_{2,-m}^{2}\left(\Omega_{P_{S} L}\right)(t)\right],
\end{aligned}
$$

where $D_{S}$ and $E_{S}$ are the axial and rhombic components of the static ZFS interaction. This part of the ZFS tensor fluctuates with respect to the laboratory frame due to overall reorientation of the molecule. The reorientational motion is modeled as isotropic rotational diffusion represented by the Liouville operator $\hat{\hat{L}}_{R}=-i D_{R} \nabla_{\Omega_{P_{S} L}}^{2}$ acting on the angle $\Omega_{P_{S} L}$ which describes the orientation of the principal axis of the static ZFS tensor $\left(P_{S}\right)$ relative to the laboratory axis $(L)$; this angle is encoded in the corresponding Wigner rotation matrices $D_{k,-m}^{2}\left(\Omega_{P_{S} L}\right), k=0,-2,2$. The $T_{m}^{2}(S)$ quantities are components of the second rank spin tensor operator and are defined as: $T_{0}^{2}(S)=1 / \sqrt{6}\left[3 S_{z}^{2}-S(S+1)\right], T_{ \pm 1}^{2}(S)$ $=\mp \frac{1}{2}\left[S_{z} S_{ \pm}+S_{ \pm} S_{z}\right]$ and $T_{ \pm 2}^{2}(S)=\frac{1}{2} S_{ \pm} S_{ \pm}$. The slow motion theory assumes that the transient ZFS, $H_{\mathrm{ZFS}}^{\mathrm{T}}(t)$, also possesses its own principal axis system $\left(P_{T}\right)$ and a constant amplitude $\Delta_{T}^{2}=\frac{2}{3} D_{T}^{2}+2 E_{T}^{2}$, where $D_{T}$ and $E_{T}$ are the axial and rhombic components of the transient ZFS, respectively. The transient ZFS defined in the $\left(P_{T}\right)$ frame, which is not fixed in the molecule (contrary to the $\left(P_{S}\right)$ frame), is transformed, first according to a pseudorotational diffusion to the $\left(P_{S}\right)$ frame (via the $\Omega_{P_{T} P_{S}}$ angle), and second by the reorientational diffusion of the entire molecule to the ( $L$ ) frame (via the $\Omega_{P_{S} L}$ angle):

$$
H_{\mathrm{ZFS}}^{T(L)}(t)=\sum_{m=-2}^{2}(-1)^{m} V_{-m}^{2 T(L)} T_{m}^{2}(S)
$$

with

$$
\begin{aligned}
V_{-m}^{T(L)}= & \sum_{n=-2}^{2}\left\{\sqrt{\frac{2}{3}} D_{T} D_{0,-n}^{2}\left(\Omega_{P_{S} P_{T}}(t)\right)\right. \\
& \left.+E_{T}\left[D_{-2,-n}^{2}\left(\Omega_{P_{S} P_{T}}\right)(t)+D_{2,-n}^{2}\left(\Omega_{P_{S} P_{T}}\right)(t)\right]\right\} \\
& \times D_{-n,-m}^{2}\left(\Omega_{P_{S} L}(t)\right) .
\end{aligned}
$$

The pseudorotational diffusion modulating the orientation $\Omega_{P_{T} P_{S}}$ of the principal axis system of the transient $\mathrm{ZFS}\left(P_{T}\right)$ 
with respect to the molecule fixed frame $\left(P_{S}\right)$ is supposed to reflect any distortional motion of the complex leading to stochastic fluctuations of the transient ZFS tensor. The pseudorotational diffusion operator (distortional operator), $\hat{\hat{L}}_{D}$, is defined as $\hat{\hat{L}}_{D}=-i D_{D} \nabla_{\Omega_{P_{T} P_{s}}}^{2}$ in full analogy to the isotropic rotational diffusion operator, $\hat{\hat{L}}_{R}$. This explains why this very simple model of the transient ZFS is referred to as the "pseudorotational model." $3,4,6,30$ The rotational and pseudorotational (distortional) diffusion constants $D_{R}$ and $D_{D}$, respectively, are related to rotational and distortional correlation times, $\tau_{R}$ and $\tau_{D}$ which refer to the corresponding second rank Wigner rotation matrices. Having explained the components of the lattice dynamics (the electron spin subsystem is treated as its part) one can rewrite Eq. (1) as a matrix product: $3,4,29,30$

$$
\begin{aligned}
K_{1,1}^{D D} & \left(-\omega_{I}\right) \\
& =\frac{1}{2 S+1}\left[T_{1}^{1(D D)}\right]^{+}\left[\hat{\hat{M}}_{\mathrm{NMRD}}\left(\omega_{I}\right)\right]^{-1}\left[T_{1}^{1(D D)}\right] .
\end{aligned}
$$

The operator $\hat{\hat{M}}_{\mathrm{NMRD}}\left(\omega_{I}\right)$ is given as $\hat{\hat{M}}_{\mathrm{NMRD}}\left(\omega_{I}\right)$ $=-i\left[\hat{\hat{L}}_{Z}\left(\omega_{S}\right)+\hat{\hat{L}}_{\mathrm{ZFS}}^{S}+\hat{\hat{L}}_{\mathrm{ZFS}}^{T}+\hat{\hat{L}}_{R}+\hat{\hat{L}}_{D}+\hat{\hat{1}} \omega_{I}\right],\left[\hat{\hat{M}}_{\mathrm{NMRD}}\left(\omega_{I}\right)\right]$ is its matrix representation, while $\left[\hat{\hat{M}}_{\mathrm{NMRD}}\left(\omega_{I}\right)\right]^{-1}$ denotes the inverted matrix. The matrix form of the $\hat{\hat{M}}_{\mathrm{NMRD}}\left(\omega_{I}\right)$ operator is set up in a basis $\left.\left\{\mid O_{i}\right)\right\}$ given as an outer product of vectors associated with the degrees of freedom of the system: $\left.\left.\left.\left.\quad\left|O_{i}\right|=\mid A B C\right) \otimes \mid L K M\right) \otimes \mid \Sigma \sigma\right),\right)^{3,4,30}$ with the distortional and rotational components $\mid A B C$ ) and $\mid L K M)$ defined as: $\mid A B C)=|A B C\rangle\left\langle A B C|=| \Psi_{B C}^{A}\right\rangle$ $=\sqrt{(2 A+1) / 8 \pi^{2}} D_{B C}^{A}\left(\Omega_{P_{T} P_{S}}\right) \quad$ and $\left.\quad \mid L K M\right)=|L K M\rangle$ $\left\langle L K M|=| \Psi_{K M}^{L}\right)=\sqrt{(2 L+1) / 8 \pi^{2}} D_{K M}^{L}\left(\Omega_{P_{S} L}\right)$, respectively, while the spin component $\mid \Sigma \sigma)$ are related to the basis vectors $\left|S, m_{S}\right\rangle\left\langle S, m_{S}^{\prime}\right|$ forming the Liouville space for the electron $\quad$ spin $\quad S: \quad \mid \Sigma \sigma)=\sum_{m}(-1)^{S-m-\sigma} \sqrt{2 \Sigma+1}$ $\left(\begin{array}{ccc}S & S & \Sigma \\ m+\sigma & -m & -\sigma\end{array}\right)|S, m+\sigma\rangle\langle S, m|$ where $\Sigma$ ranges from 1 to $2 S$. The vector $\left[T_{1}^{1(D D)}\right]$ represents the electron spin tensor operator contributing to the coupling between the nuclear spin $I$ and the lattice, in this case the nuclearelectron dipole-dipole coupling: $H_{I L}=H^{(D D)}(I, S)$ $=\sum_{n=-1}^{1}(-1)^{n} I_{n}^{1} T_{-n}^{1(D D)}$. 3,4 For the inner-sphere PRE the $T_{1}^{1(D D)}$ operator has the form: $T_{1}^{1(D D)}=a_{D D}^{I S}$ $\left.\sqrt{5(2 S+1)(S+1) S / 3} \sum_{q=-1}^{1}\left(\begin{array}{ccc}2 & 1 & 1 \\ 1-q & q & -1\end{array}\right) \mid 1, \sigma\right) D_{0,1-q}^{2}\left(\Omega_{D D L}\right)$, with $a_{D D}^{I S}=\sqrt{6} \frac{\mu_{0}}{4 \pi} \frac{\gamma_{I} \gamma_{S} \hbar^{2}}{r_{\text {IS }}^{3}}\left(r_{\text {IS }}\right.$ denotes the nuclear-electron interspin distance, other symbols have the usual meaning). This implies that the vector $\left[T_{1}^{1(D D)}\right]$ contains three non-zero elements corresponding to the states |000)|202)|1-1), $\mid 000)|201| 10$ ), and |000)|200)|11). This means, in turn, that a $3 \times 3$ fragment of the inverted supermatrix $\left[\hat{\hat{M}}_{\mathrm{NMRD}}\left(\omega_{I}\right)\right]^{-1}$ is needed to calculate the nuclear spin relaxation rate, $R_{1 I}^{\mathrm{PRE}}\left(\omega_{I}\right)$, as illustrated in Fig. 1. The above expression for the $T_{1}^{1(D D)}$ operator contains the angle $\Omega_{\mathrm{DDL}}$ describing the orientation of the nuclear spin-electron spin dipole-dipole axis with respect to the laboratory frame. In general, the dipole-dipole axis does not coincide with the $\left(P_{S}\right)$ frame; a set of time independent angles, $\Omega_{\mathrm{DDP}_{S}}$, is incorporated

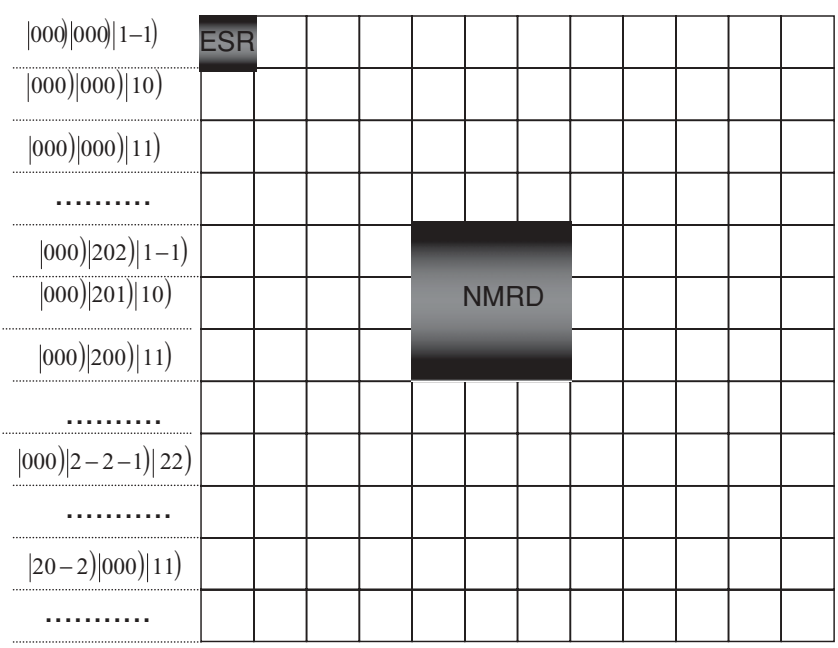

FIG. 1. Structure of the supermatrix $\left[M_{\mathrm{ESR}}\right]$ ([$\left.\left.M_{\mathrm{NMRD}}\right]\right)$. ESR spectra are represented by the $\mid 000)|000| 1-1)$ element of the inverted supermatrix, while NMRD are given by its $3 \times 3$ block associated with the states: |000)|202)|1 - 1), |000)|201|10), and |000)|200)|11).

into the slow motion theory to account for this effect. The measured nuclear spin relaxation of water protons, $R_{1 I}\left(\omega_{I}\right)$, in solutions of paramagnetic complexes is given as $R_{1 I}\left(\omega_{I}\right)=P_{M} q /\left[\left(R_{1 I}^{\mathrm{PRE}}\right)^{-1}+\tau_{M}\right]+R_{1 I}^{\text {outer }}\left(\omega_{I}\right)$, where $q$ is the number of bound water molecules, $P_{M}$ is the mole fraction of water protons in bound position, $\tau_{M}$ is the mean residence lifetime of the bound water molecule (exchange lifetime). The exchange lifetime also affects the $R_{1 I}^{\mathrm{PRE}}\left(\omega_{I}\right)$ and has to be included into the diagonal terms of the $\left[\hat{\hat{M}}_{\mathrm{NMRD}}\left(\omega_{I}\right)\right]$ matrix as $\tau_{M}^{-1}$. Calculations of the outer-sphere contribution, $R_{1 I}^{\text {outer }}\left(\omega_{I}\right)$, are considerably more demanding. The outer-sphere slow motion theory was introduced by Kruk and Kowalewski. ${ }^{38}$ Since the principal concept is the same, we do not discuss here the outer-sphere version of the slow motion theory.

The slow motion approach can in a straightforward manner be adapted to describe ESR spectral lineshapes.

\section{B. Slow motion theory in application to ESR}

An ESR lineshape function $L\left(\omega_{S}-\omega\right)$ is determined by the spectral density $s_{-1,-1}(\omega)$ $=\int_{0}^{\infty}\left\langle S_{-1}^{+}(\tau) S_{-1}(0)\right\rangle \exp (-i \omega \tau) d \tau \quad\left(L\left(\omega_{S}-\omega\right) \propto\right.$ $\left.s_{-1,-1}(\omega)\right)$ corresponding to the single-quantum transitions of the electron spin. ${ }^{25,42}$ Thus, derivations of the ESR lineshapes follow the same procedure as that of NMRD, described in Sec. II A. The lineshape function is given as:

$$
\begin{aligned}
L\left(\omega_{S}-\omega\right)= & \int_{0}^{\infty} \operatorname{Tr}_{S}\left\{S_{-1}^{1+}\left[\exp \left(-i \hat{\hat{L}}_{S} \tau\right) S_{-1}^{1}\right] \rho_{S}^{e q}\right\} \\
& \times \exp (-i \omega \tau) d \tau \propto\left[S_{-1}^{1}\right]^{+}\left[\hat{\hat{M}}_{\mathrm{ESR}}(\omega)\right]^{-1}\left[S_{-1}^{1}\right] .
\end{aligned}
$$

The superoperator $\hat{\hat{M}}_{\mathrm{ESR}}(\omega)$ contains the same terms (originating from the spin Hamiltonian model) as $\hat{\hat{M}}_{\mathrm{NMRD}}\left(\omega_{I}\right)$ :

$$
\begin{aligned}
& \hat{\hat{M}}_{\mathrm{ESR}}(\omega) \\
& \quad=-i\left[\hat{\hat{L}}_{Z}(S)+\hat{\hat{L}}_{\mathrm{ZFS}}^{S}+\hat{\hat{L}}_{\mathrm{ZFS}}^{T}+\hat{\hat{L}}_{R}+\hat{\hat{L}}_{D}+\hat{\hat{1}} \omega\right] .
\end{aligned}
$$


The only change is the term $\hat{\hat{1}} \omega$ which now replaces the term $\hat{\hat{1}} \omega_{I}$. The nuclear spin relaxation is described by a spectral density taken at the nuclear Larmor frequency $\omega_{I}$, while the electron spin transitions determining the ESR lineshape occur at three orders of magnitude larger frequencies $\omega$. The vector $\left[S_{-1}^{1}\right]$ contains expansion coefficients of the tensor operator $S_{-1}^{1}$ in the basis $\left.\left\{\mid O_{i}\right)\right\}$. In fact, there is just one nonzero coefficient, namely the one associated with the basis vector $\mid A B C) \mid L K M) \mid \Sigma \sigma)=\mid 000) \mid 000) \mid 1-1)$. Thus the ESR lineshape is determined by one element of the inverted matrix $\left[\hat{\hat{M}}_{\mathrm{ESR}}(\omega)\right]^{-1}$, as shown in Fig. 1 .

The slow motion theory of ESR lineshape obviously is also valid for arbitrary interaction strengths and rates of isotropic reorientation. Moreover, the theory includes in a natural way phenomena, such as cross-correlation effects and dynamic frequency shifts.

Even though one cannot explicitly define the electron spin relaxation operator (and, in consequence, electron spin relaxation times), the spectral density function $s_{-1,-1}(\omega)$ describing the lineshape has a well-defined meaning.

\section{Slow motion theory for ESR in high spin systems and g-tensor anisotropy}

In discussions of electron spin relaxation and ESR lineshapes for $S=1 / 2$ systems, one usually considers the effects of the anisotropy of the $g$-tensor and of the hyperfine interaction. ${ }^{22,26}$ Sometimes, one also invokes the spin-rotation interaction. ${ }^{23,48}$ In high-spin systems, these interactions are also present. In small gadolinium (III) complexes, the spin-rotation mechanism was included at an early stage, ${ }^{48,58}$ but discarded in more recent work. ${ }^{19-22}$ The hyperfine interaction with magnetic gadolinium isotopes $\left({ }^{155} \mathrm{Gd}\right.$, ${ }^{157} \mathrm{Gd}$ ) appears to be of little importance, ${ }^{59}$ but the $g$-tensor anisotropy may play a role, in particular at high magnetic fields. It is possible to include the $g$-anisotropy effects into the slow motion formulation of this work by modifying the form of the Zeeman Hamiltonian and the corresponding Liouvillian. The details of this modification are described in the appendix. The price to pay for this extension (which amounts to lowering the symmetry of the problem) is that the superoperator $\hat{\hat{M}}_{\mathrm{ESR}}$ in Eq. (6) will have a significantly larger number of non-vanishing elements in the $\mid \mathrm{ABC}) \mid \mathrm{KLM}) \mid \Sigma \sigma)$ basis. In order not to let this problem become unwieldy, we choose here to limit ourselves to the case of cylindrically symmetric $g$-tensor, i.e., to the case $g_{x x}=g_{y y}$ $\neq g_{z z}$, where $g_{x x}, g_{y y}, g_{z z}$ are principal cartesian components of the $g$-tensor. Moreover, we set $g_{\text {eff }}=\frac{1}{3}\left(g_{x x}+g_{y y}+g_{z z}\right)$ $=2$. The $g$-tensor anisotropy is thus represented by a single parameter: $\Delta g=g_{\text {eff }}-g_{z z}$. In addition, it is assumed that the principal axis system of the $g$-tensor coincides with the $\left(P_{S}\right)$ frame (if $\Omega_{D D P_{S}}=0$, it means that the dipole-dipole axis, the $\left(P_{S}\right)$ frame and the $g$-tensor principal $z$-axis coincide). The expressions for the matrix elements of the Zeeman Liouvillian for this form of the $g$-tensor are given in the Appendix.

In the next section we apply the ESR and NMRD slow motion approach to experimental data collected for deriva- tives of DOTA-Gd complexes of the electron spin quantum number $S=7 / 2$.

\section{UNIFIED ANALYSIS OF NMRD AND ESR DATA}

In order to verify and discuss the concept of the electron spin dynamic modeled by means of the transient ZFS modulated by a distortional motion according to the isotropic (pseudo)rotational model, we attempt in this section a consistent interpretation of NMRD profiles and ESR lineshapes for two $\mathrm{Gd}^{3+}$ complexes denoted as P760 and P792. The complexes were a gift of Guerbet. The complexes are hydrophilic derivatives of DOTA-Gd, with molecular masses of 5.6 and $6.5 \mathrm{kDa}$, respectively. The NMRD data have been taken from Vander Elst et al. ${ }^{60,61}$ for P760 and P792, respectively. In both cases the relaxation data were collected at $310 \mathrm{~K}$. The concentration of P760 was $0.19 \mathrm{mMol}$, while for P792 two concentrations were used, 0.285 and $1 \mathrm{mMol}$. ESR lineshapes for corresponding concentrations of both complexes were measured at two frequencies, 95 and $237 \mathrm{GHz}$ (additionally, ESR measurements at $9 \mathrm{GHz}$ were attempted. However, the signal was very broad and difficult to extract from the base-line, so it was not used) and two temperatures: 310 and $290 \mathrm{~K}$. The ESR experiments were carried out at ACERT (National Biomedical Center for Advanced ESR Technology, Cornell University). The effects of changing the temperature on the ESR spectra were small and we limit ourselves in most cases to presenting the data at $310 \mathrm{~K}$.

Before embarking on the combined analysis of the NMRD and ESR data, we wish to report some experimental ESR observations for the two Gd(III) complexes, used as model systems in this study, obtained in the solid state. We have taken $237 \mathrm{GHz}$ ESR spectra of the solid powder (at room temperature) of the complex denoted as P760 and of the frozen solution of the complex P792 (at $193 \mathrm{~K}$ ). In both these cases, molecular reorientations are suppressed and the observed lineshapes should correspond to powder patterns. The spectra, shown in Fig. 2(a) (P760) and Fig. 2(b) (P792), are very narrow and the lines are symmetric. If one neglects the (static) ZFS effects, then the lineshapes are consistent with a slightly anisotropic $g$-tensor of non-axial symmetry, close to the limit $g_{x x}-g_{y y}=g_{y y}-g_{z z}$. For the P760 powder, the shape can be reproduced with $g_{x x}-g_{y y}=g_{y y}$ $-g_{z z}=0.0007 \pm 0.0003$, while for the solid solution of P792 we obtain the upper bound of the $g$-anisotropy of around 0.0008-0009. However, the assumption of the negligible ZFS effects in the solid samples of Gd(III) complexes does not seem realistic. Indeed, Benmelouka et al. ${ }^{56,62}$ reported lowtemperature, high-field ESR spectra for P792, in the powder and frozen solution forms, which were possible to interpret based only on the ZFS effects. The fact that the lines in Figs. 2(a) and 2(b) are so narrow may perhaps indicate the occurrence of some form of dynamic phenomena.

Turning to the main topic of this work, the interpretation of nuclear relaxation data requires more parameters than ESR lineshapes. This is a consequence of a "hierarchy of events" in paramagnetic systems: electron spin dynamics is almost unaffected by the presence of nuclear spins (in fact, such an effect is completely neglected in the present theoretical description), 


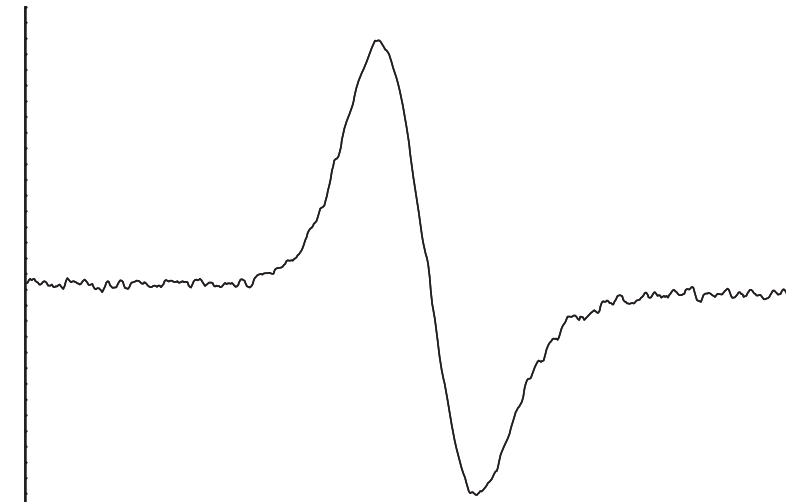

(a)

\begin{tabular}{lllllll}
\hline 8.46 & 8.47 & 8.48 & 8.49 & 8.50 & 8.51 & 8.52
\end{tabular}

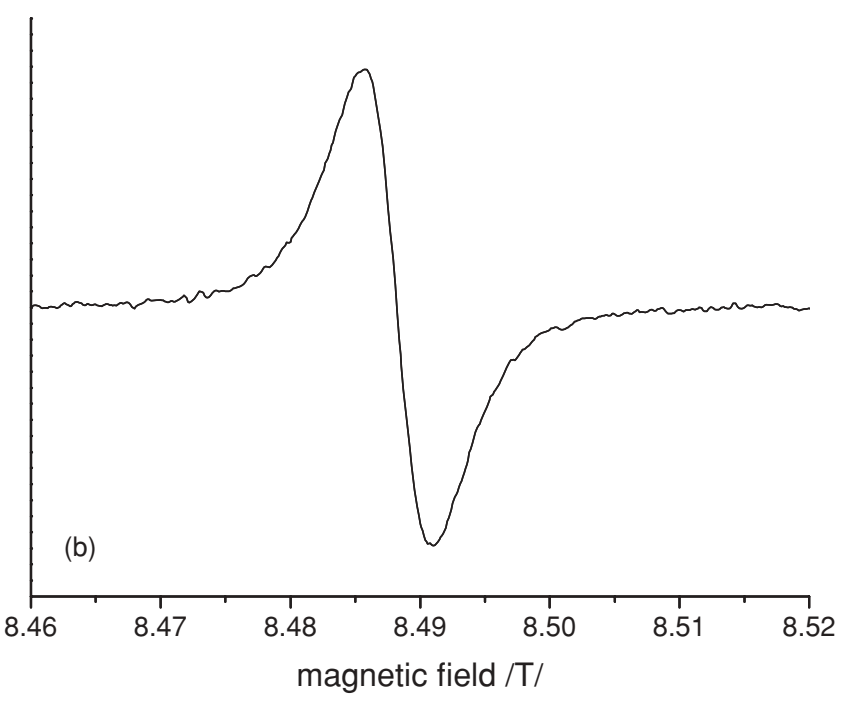

FIG. 2. $237 \mathrm{GHz}$ ESR spectra of (a) the solid powder (at room temperature) of P760, (b) the frozen solution of the complex P792 (at $193 \mathrm{~K}$ ).

while the nuclear spin relaxation is very considerably affected by the properties of the electron spin subsystem. Thus, besides the static and transient ZFS amplitudes, $D_{S}$ and $D_{T}$, the rotational correlation time $\tau_{R}$ and the distortional correlation time $\tau_{D}$, which according to the applied model fully determine the ESR spectra, the inner-sphere nuclear relaxation profiles are influenced by the exchange life time $\tau_{M}$ and the electron-spin-nuclear spin distance scaling the relaxation profile as $r_{I S}^{-6}$. Exact interspin distances for these complexes are not available. The nuclear spin relaxation for the aqueous protons can also be caused by the outer-sphere mechanism, which in the simplest description depends on the mutual translational diffusion coefficient and the distance of closest approach of the nuclear and electron spins.

The exchange life time has been independently estimated for both complexes by means of ${ }^{17} \mathrm{O}$ relaxometry, and the obtained values are within ranges $\tau_{M}=100-140 \mathrm{~ns}$ (Ref. 60) and $\tau_{M}=80-100$ ns (Ref. 61) for P760 and P792, respectively, depending on the applied fitting strategy. In addition, the reorientational correlation times were estimated as being about $\tau_{R}=2.2 \mathrm{~ns}$ (from ${ }^{2} \mathrm{H}$ relaxation data) and $\tau_{R}=1.8 \mathrm{~ns}$ (from Stokes-Einstein equation using the molecular volume from photon correlation spectroscopy) for P760, ${ }^{60}$ and $\tau_{R}$ $=2.4 \mathrm{~ns}$ (from relaxation rates of ${ }^{17} \mathrm{O}$ ) for P792. ${ }^{61}$ Analyzing the NMRD data for both complexes in terms of inner- and outer-sphere contributions, the authors obtained $\tau_{R}=1.8 \mathrm{~ns}$ (for $\tau_{M}=320 \mathrm{~ns}$ ) for P760 (Ref. 60) and $\tau_{R}=1.7 \mathrm{~ns}$ (for $\tau_{M}=96 \mathrm{~ns}$ ) for P792. ${ }^{61}$ The description of the nuclear relaxation used in both papers ${ }^{60,61}$ was based on the classical Solomon-Bloembergen-Morgan ${ }^{2-4}$ formula for the innersphere and on the outer-sphere model proposed by Hwang and Freed. ${ }^{39}$

We choose to start the analysis from the NMRD profile for P760. Even though NMRD data need more parameters, in this case the reorientational correlation time, $\tau_{R}$, and the exchange lifetime, $\tau_{M}$, have been independently estimated. The advantage of starting with the NMRD is not only our previous experience (the slow motion theory has been already used to interpret several relaxation profiles, the work by Kowalewski et al. is a recent example ${ }^{63}$ ), but also possible difficulties in interpreting in a consistent way the ESR spectra collected at two frequencies.

First, we attempted to fit the NMRD profile including only the inner-sphere contribution and keeping the exchange life time and the rotational correlation time fixed to $\tau_{M}=120 \mathrm{~ns}$ and $\tau_{R}=1.8 \mathrm{~ns}$. The amplitudes of the static and transient ZFS, the distortional correlation time and the interspin distance were treated as adjustable parameters. Fig. 3 shows the result of the least squares fits. The first set of obtained parameters is given in Table I, case A. Using it, one can estimate the outer-sphere contribution setting the translational diffusion coefficient, $D_{\text {Diff, }}$ to the water diffusion coefficient at $310 \mathrm{~K}, D_{\text {Diff }}=3.05 \times 10^{-9} \mathrm{~m}^{2} / \mathrm{s}$, and the distance of closest approach to $d=3.7 \AA$ (this seems to be a reasonable value taking into account that $r_{I S}=3.2 \AA$ ). The outer-sphere contribution is also shown in Fig. 3. To emulate

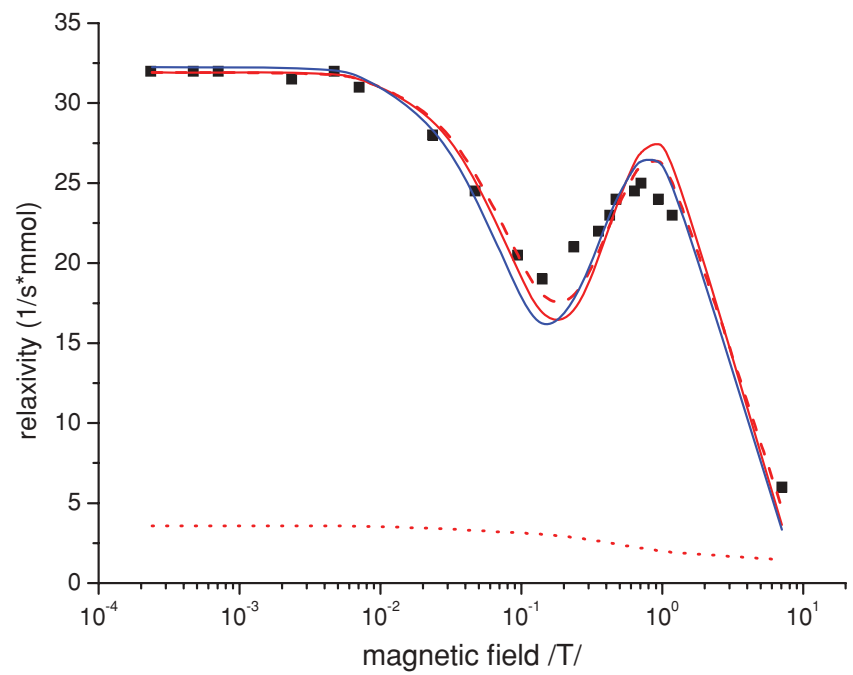

FIG. 3. Experimental NMRD profile for P760 at $310 \mathrm{~K}$ (Ref. 58) (solid squares) and the result of least square fits: solid red line (parameters given in Table I as case A), rel.err. $=0.62$; dotted red line shows a contribution of the outer-sphere relaxation mechanism calculated for the same parameters and $D_{\text {Diff }}=3.05 \times 10^{-9} \mathrm{~m}^{2} / \mathrm{s}, d=3.7 \AA$. Dashed red line shows a sum of the outer-sphere and inner-sphere contributions rescaled by the factor 0.89 that corresponds to $r_{I S}=3.27 \AA$, rel. err. $=0.49$. Solid blue line: case B parameters (inner-sphere only), rel.err. $=0.47$. 
TABLE I. Different parameter sets used to simulate NMRD data and ESR lineshapes for P760 (cases A-F) and P792 (cases G-I). Parameters given in parentheses were kept fixed when fitting the NMRD profiles. Parameters in bold correspond to overall best fits for the two complexes.

\begin{tabular}{|c|c|c|c|c|c|c|c|}
\hline \multirow[b]{3}{*}{ Case } & \multicolumn{6}{|c|}{ Parameters affecting NMRD } & \multirow{3}{*}{$\begin{array}{l}\text { Not important for NMRD } \\
\qquad \Delta g=g_{e f f}-g_{z z}\end{array}$} \\
\hline & \multicolumn{2}{|c|}{ Not important for ESR } & \multicolumn{4}{|c|}{ Parameters affecting ESR lineshapes } & \\
\hline & $\tau_{M}(\mathrm{~ns})$ & $r_{I S}(\mathrm{pm})$ & $D_{S}\left(10^{-2} \mathrm{~cm}^{-1}\right)$ & $D_{T}\left(10^{-2} \mathrm{~cm}^{-1}\right)$ & $\tau_{R}(\mathrm{~ns})$ & $\tau_{D}(\mathrm{ps})$ & \\
\hline A & $(120)$ & 320 & 5.26 & 2.02 & $(1.8)$ & 14.9 & 0/0.0016/0.0018 \\
\hline B & $(120)$ & 325 & 4.34 & 1.76 & (1.8) & 15.6 & $0 / 0.0015$ \\
\hline $\mathrm{C}$ & $(120)$ & 285 & 4.51 & 2.5 & 0.64 & 39.7 & 0/0.0025/0.009 \\
\hline $\mathrm{D}$ & $(120)$ & 319 & 4.59 & 1.78 & 1.36 & 18.9 & 0/0.0015/0.007 \\
\hline $\mathrm{E}$ & 137 & 327 & 4.18 & 1.62 & $(1.8)$ & 16.9 & $0 / 0.0015$ \\
\hline $\mathrm{F}$ & 103 & 319 & 4.53 & 1.69 & 1.77 & $\mathbf{5 7 . 8}$ & $0 / \mathbf{0 . 0 0 1 5}$ \\
\hline G & 75 & 318 & 2.4 & 1.22 & $(1.8)$ & 68.9 & \\
\hline $\mathrm{H}$ & (96) & 318 & 4.15 & 1.76 & 2.9 & 47.7 & \\
\hline I & 138 & 316 & 4.03 & 1.54 & 3.2 & 40.8 & 0/0.0005/0.0008/0.0013 \\
\hline
\end{tabular}

a complete relaxation profile, containing the inner- and outersphere parts and still being in a reasonable agreement with the experimental data, we have "rescaled" the inner-sphere contribution by setting a longer interspin distance, $r_{I S}=3.27 \AA$, and compensating the effect with the outer-sphere contribution. The resulting calculated curve is also shown in Fig. 3. Starting from somewhat different values (but still keeping $\tau_{M}$ $=120 \mathrm{~ns}, \tau_{R}=1.8 \mathrm{~ns}$ ) one gets another fitted curve (the final parameters, displayed in Table I, case B, do not very significantly differ from the previous ones), shown in Fig. 3 as well. Since the first set of parameters (after including the outersphere contribution) and the second one lead to fits of similar quality, we shall discuss the ESR lineshapes for both cases.

The ESR lineshape, predicted for both frequencies by the pseudorotational model for the case A parameters, without and with $g$-anisotropy effects, are shown in Figs. 4(a) and 4(b) and compared with the experimental results for $310 \mathrm{~K}$. Excluding the $g$-anisotropy, the theoretical lineshape at 237 $\mathrm{GHz}$ is somewhat too narrow compared to the experimental one, yet on the whole, we judge the agreement as acceptable. The calculated ESR spectra have been treated as given in arbitrary units, i.e., we scale them (multiply by a number which is different for every spectrum) to get the best possible agreement with the experiment. At the lower frequency $(95 \mathrm{GHz})$, panel $b$, the discrepancies between the theoretical predictions and the experimental spectrum are, at the first glance, more significant.

One should remember that the ESR spectrum is a derivative of the ESR absorption line. Thus, one should realize that the calculated spectrum displayed in Fig. 4(b) does not exhibit "two lines" on the right side, in contrary to the experiment. The right part of the theoretical spectrum only reflects a different monotonic decay of a theoretical absorption line compared to the experimental one. One can see that from the inset, in which an example of an integrated spectrum is presented. The ESR spectra were collected at high magnetic fields compared to the NMRD profiles. The analysis performed so far indicates that the general difficulties with a unified analysis of the NMRD and ESR data are due to slow electron spin relaxation predicted by the theory. The calculated spectrum for the higher magnetic field is too narrow, while the spectrum for the lower magnetic field shows features resulting from the static ZFS which would also be less pronounced for a faster electron spin dynamics. A possible solution of this problem is to allow for effects of increasing influence of electron $g$-tensor anisotropy for high magnetic fields.

As one can see from Fig. 4(a), a relatively small $g$ tensor anisotropy, $\Delta g=0.0018$, leads to a sufficient broadening of the ESR spectrum at the high magnetic field. This rather small $g$-tensor anisotropy considerably influences the shape of the ESR spectrum at $95 \mathrm{GHz}$, reducing the effects of the static ZFS. The inset actually illustrates how significantly the $g$-tensor anisotropy affects the absorption ESR line. The $g$-tensor anisotropy of this magnitude seems to be justified when compared to the literature values for $\mathrm{Gd}(\mathrm{III}){ }^{64,65}$ The anisotropy of the $g$-tensor influences also NMRD profiles. However, taking into account the range of NMRD magnetic fields, the $g$-tensor anisotropy can safely be neglected for all but perhaps the highest field point of the relaxation profiles of both complexes. An extension of NMRD slow motion theory by including $g$-tensor anisotropy interaction in all its details is a very complicated task from the computational point of view. The difficulties are caused by the fact that the $g$-tensor anisotropy leads, besides affecting the electron spin dynamics, to additional terms in the electron spin nuclear spin dipole-dipole coupling. ${ }^{66,67}$ In consequence, to get a complete description of all the effects relevant for the nuclear spin relaxation, one has to set up a very large matrix $\hat{\hat{M}}$. Its dimension for higher spin quantum numbers exceeds the size allowed by the FORTRAN compiler. At this stage of developing the software we have not solved this problem yet.

A question which appears in the course of this analysis is about possible effects of static ZFS rhombicity on the ESR lineshape. The role of the rhombic terms in the case of NMRD has been rather intensively discussed in the literature. ${ }^{18,68,69}$ One can see from Fig. 4(b) that the rhombicity of the static ZFS does not lead to significant changes of the ESR lineshape. Therefore, we do not discuss this issue any further. Summarizing, one can say at this stage that the set of parameters denoted as case A (with $\Delta g=0.0018$ ), gives an acceptable interpretation of the multifrequency ESR lineshapes and the NMRD data. 

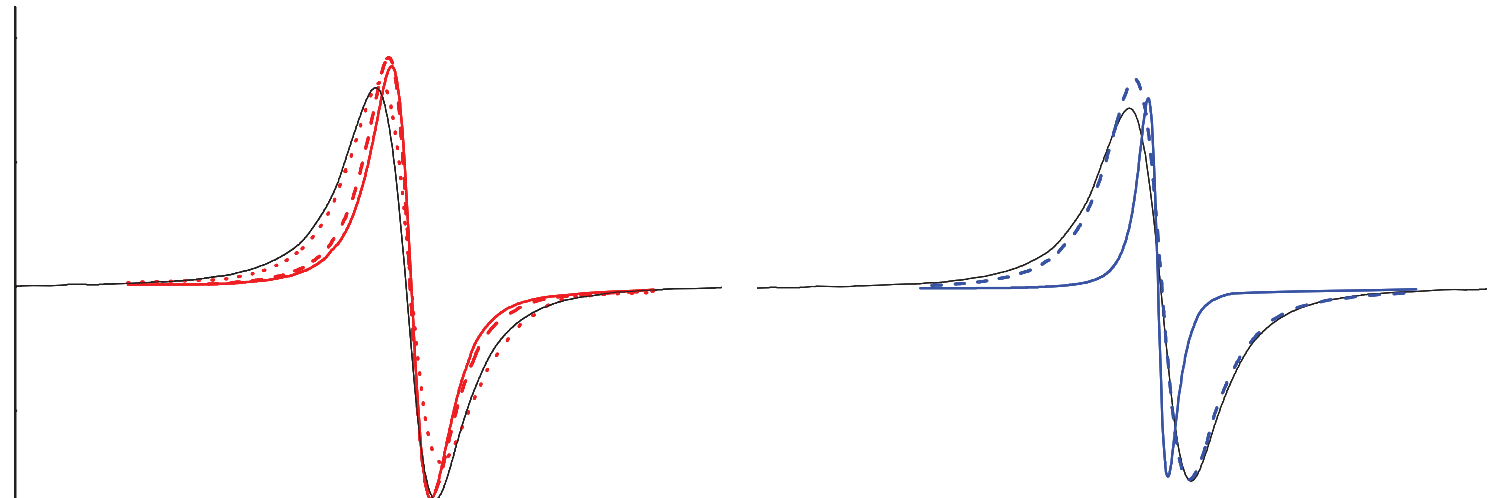

(a)

(c)

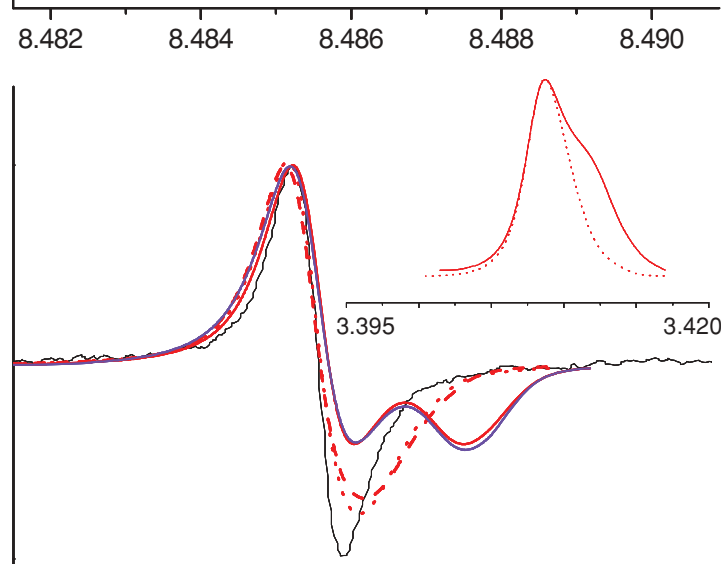

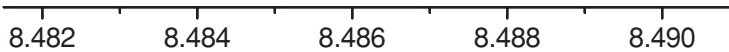

(b)

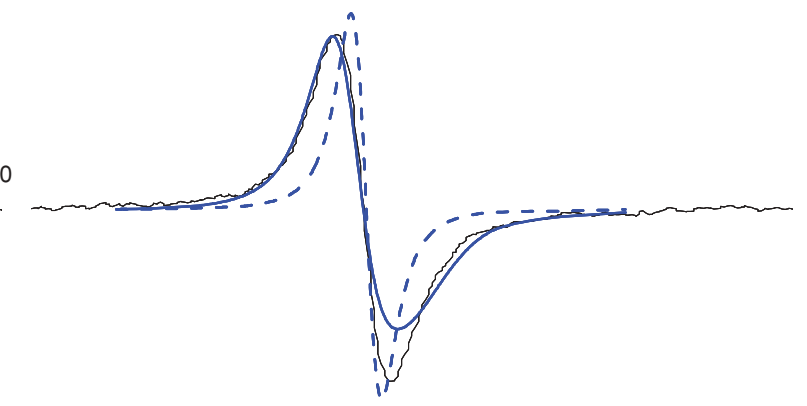

(d)

\begin{tabular}{|c|c|c|c|}
\hline 3.400 & 3.405 & 3.410 & 3.415 \\
\hline
\end{tabular}

$\begin{array}{cccc}3.400 & 3.405 & 3.410 & 3.415 \\ & \text { magnetic field /T/ }\end{array}$

FIG. 4. Experimental ESR lineshape for $0.19 \mathrm{mMol}$ P760 at $310 \mathrm{~K}$ (black solid line) for (a, c) $237 \mathrm{GHz}$ and (b, d) $95 \mathrm{GHz}$. (a, b) Theoretical ESR lineshapes for the case A parameters and $\Delta g=0$ (red solid line), $\Delta g=0.0016$ (red dashed line), $\Delta g=0.0018$ (red dotted line). The purple line in (b) shows the theoretical ESR lineshape obtained for this set of parameters $(\Delta g=0)$, allowing for a rhombic static ZFS, $E_{S} / D_{S}=0.1$. Inset - integrated theoretical spectra corresponding to the case A parameters with $\Delta g=0$ (solid red line) and $\Delta g=0.0018$ (dotted red line). (c, d) Theoretical ESR lineshape for the case B parameters with $\Delta g=0$ (blue solid line), $\Delta g=0.0015$ (blue dashed line).

The ESR spectra calculated for the second set of parameters, case B in Table I, are shown in Figs. 4(c) and 4(d). Also in this case the high-field spectrum is apparently too narrow, which again suggests an additional electron spin relaxation mechanism. Again, a $g$-tensor anisotropy contribution, $\Delta g=0.0015$, leads to an appropriate broadening of the spectrum [Fig. 4(c)]. More significant discrepancies are observed for $95 \mathrm{MHz}$ [Fig. 4(d)]. It is interesting to notice that, for $\Delta g=0$, the ESR lineshape shows features caused by the static ZFS, and that the $g$-tensor anisotropy leads, by reducing these features, to an apparent narrowing of the ESR line (the lineshape becomes more Lorentzian-like). A further increase of the $g$-tensor anisotropy gives a broader ESR line, so that the proper linewidth at high field can be reached, but then the ESR spectrum at the lower magnetic field becomes too broad. In our opinion, it is rather difficult to resolve which set of parameters discussed so far (case A and case B in Table I) leads to a better agreement with the experimental data.

As it has already been discussed, the estimation of the reorientational correlation time, $\tau_{R}$, is not exact. Depending on the way of estimating it, longer $\tau_{R}$ values have been mentioned (up to even $3.4 \mathrm{~ns}$ ). ${ }^{60}$ In addition, the overall rotational motion of the complex is modeled as isotropic reorientation. Taking into account the non-spherical molecular shape of this complex, such a description might be too restrictive. Therefore, in the next step we included the (effective) rotational correlation time, $\tau_{R}$, into the set of adjustable parameters and performed a five-parameter fit $\left(D_{S}\right.$, $D_{T}, \tau_{D}, \tau_{R}$, and $r_{I S}$ ) of the NMRD profile (the exchange lifetime still being kept at $\tau_{M}=120 \mathrm{~ns}$ ). Also in this case, two considerably different sets of parameters have been obtained, $c f$. cases $\mathrm{C}$ and $\mathrm{D}$ in Table I. The results are presented in Fig. 5; note that the figure also contains results of other types of fittings, to be discussed below. It seems that the rotational correlation time, $\tau_{R}=0.64 \mathrm{~ns}$, (case $\mathrm{C}$ ) is too short; the same concerns the interspin distance, $r_{I S}=2.85 \AA$. Further, it is worth noticing that in this case the agreement with the experimental data can be significantly improved by including the outer-sphere contribution, which is relatively larger for faster molecular tumbling. ${ }^{38}$ Therefore, it is a large advantage if the analysis of NMRD profiles for paramagnetic compounds can be supported by independent estimations of as many 


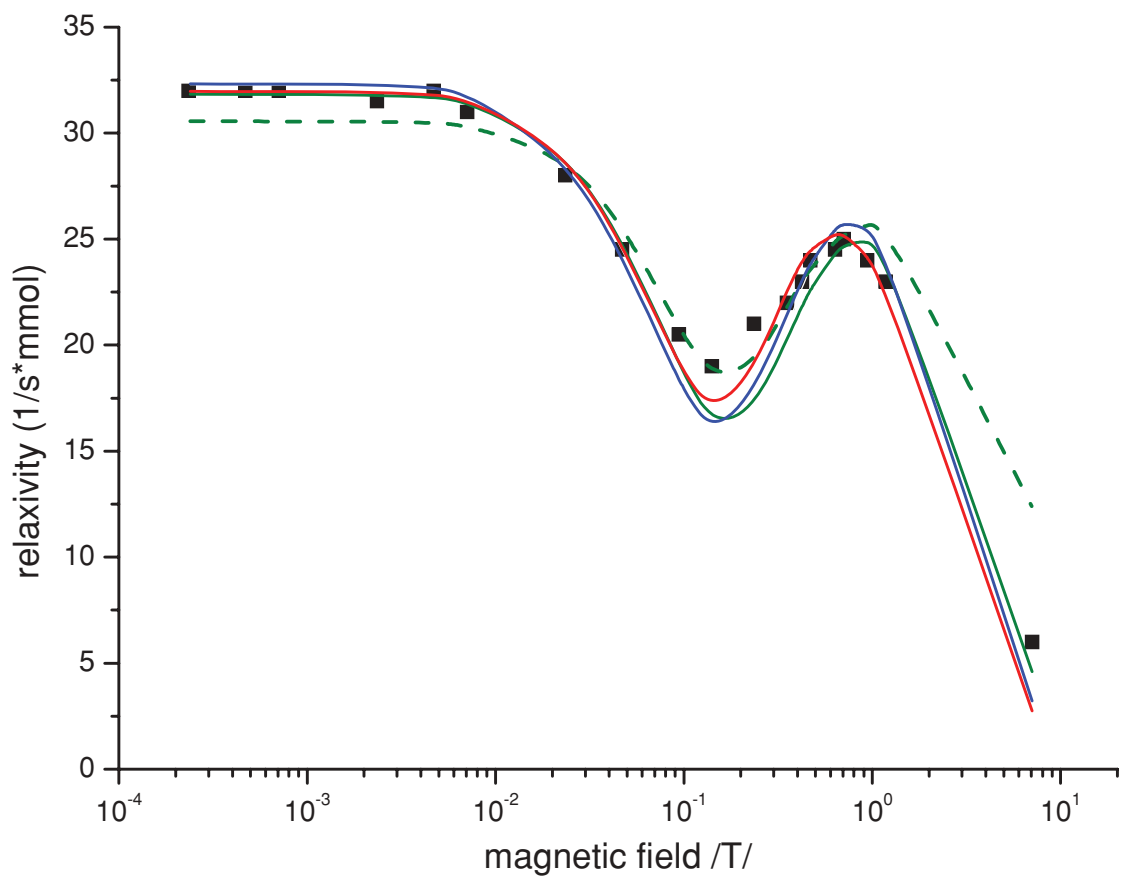

FIG. 5. Experimental NMRD profile for P760 at $310 \mathrm{~K}$ (Ref. 58) (solid squares) and the result of least square fits. Green dashed line: case C, rel.err. $=0.54$; green solid line: case D in Table I, rel.err. $=0.32$; blue solid line: case E, rel.err. $=0.35$; red solid line: case F, rel.err. $=0.22$.

parameters as possible. The crucial test for the parameters is whether they lead to a reasonable agreement with not only NMRD data but also with ESR spectra. This was tested for the cases $C$ and D sets of parameters. Indeed, as it could be predicted, the case $\mathrm{C}$ parameters do not give a proper ESR. The ESR spectrum calculated for the higher magnetic field, without allowing for $g$-tensor anisotropy, is definitely too narrow [Fig. 6(a)]. The ESR line reaches the proper linewidth when the $g$-tensor anisotropy of $\Delta g=0.0025$ is included. However, the $g$-tensor anisotropy, $\Delta g=0.009$, required for the ESR spectrum at $95 \mathrm{GHz}$ (which is also much too narrow for $\Delta g=0$, compared to the experimental one) to get the adequate broadening in Fig. 6(b) is definitely not acceptable. This means that one cannot satisfactorily reproduce the ESR spectra within this set of parameters and that the case $C$ parameters should be excluded.

The other set of parameters obtained from the 5parameter fit $\left(D_{S}, D_{T}, \tau_{D}, \tau_{R}\right.$, and $\left.r_{I S}\right)$ of the NMRD profile is denoted case $\mathrm{D}$ in Table I. Before discussing the corresponding ESR lineshape simulations, we turn back for a little while to Fig. 5. Since not only the values of the rotational correlation time, $\tau_{R}$, but also of the exchange lifetime, $\tau_{M}$, given by Vander Elst et al.. ${ }^{60}$ should be treated as approximate, we have attempted to fit the relaxation data by performing a different 5-parameter fit $\left(D_{S}, D_{T}, \tau_{D}, \tau_{M}\right.$, and $\left.r_{I S}\right)$ with fixed $\tau_{R}$ and adjustable $\tau_{M}$, and, finally, a 6-parameter fit allowing for adjustment of all relevant parameters. The results of both these fits are summarized in Table I, cases E and F, respectively, and shown in Fig. 5. The ESR spectra calculated for the case $\mathrm{D}$ and $\mathrm{E}$ parameter sets resulting from the 5-parameter fits (with fixed $\tau_{M}$ or alternatively with fixed $\tau_{R}$ ), shown in Figs. 6(c) and 6(d), are rather similar.

While the calculated ESR lines at $95 \mathrm{GHz}$ agree well with the experimental spectrum, the lines for the higher magnetic field are too narrow. This again implies the need for introducing the $g$-tensor anisotropy relaxation mechanism. One can see from Fig. 6(c) that $\Delta g=0.0015$ yields a sufficient change of the theoretical lineshape at high field. However, for the lower magnetic field we again face the same problem as in the case shown in Fig. 4(b): faster electron spin dynamics reduces the effect of the static ZFS, i.e., the line becomes narrower when taking a more lorentzian form; it is shown in Fig. 6(d) for $\Delta g=0.0015$. A further increase of the $g$-tensor anisotropy successively leads to a broadening of the ESR line [the case of $\Delta g=0.007$ is shown in Fig. 6(d)], but the $\Delta g$ values appropriate for the ESR spectrum at the higher magnetic field are too large for the spectrum at $95 \mathrm{GHz}$ and definitely too large compared to the estimated value. Therefore, when ending up with $\Delta g=0.0015$, the quality of the unified fits of the ESR spectra and the NMRD data is comparable with the case presented in Figs. 4(a) and 4(b). In other words, extending the number of fitted parameters in the analysis of NMRD profile from four to five does not give much overall improvement.

The 6-parameter fit of the NMRD profile (case F in Table I, also presented in Fig. 5) leads to a very good agreement with the experimental NMR relaxation data. Compared to the previous results, in this case the distortional correlation time, $\tau_{D}=57.8 \mathrm{ps}$, is significantly longer. In view of the simplicity of the pseudorotation model, the physical significance of this parameter (decay constant of an assumed single exponential correlation function) should not be exaggerated. It is worth to notice that the quality of this fit is significantly better than of the fits presented by Vander Elst et al. ${ }^{60}$ and, in addition, the obtained $\tau_{M}$ value agrees now with the one estimated from independent measurements, while a much larger value $(360 \mathrm{~ns})$ was reported in the previous study. ${ }^{60}$ The ESR spectra calculated for this set of parameters (Fig. 7) and $\Delta g=0$ 


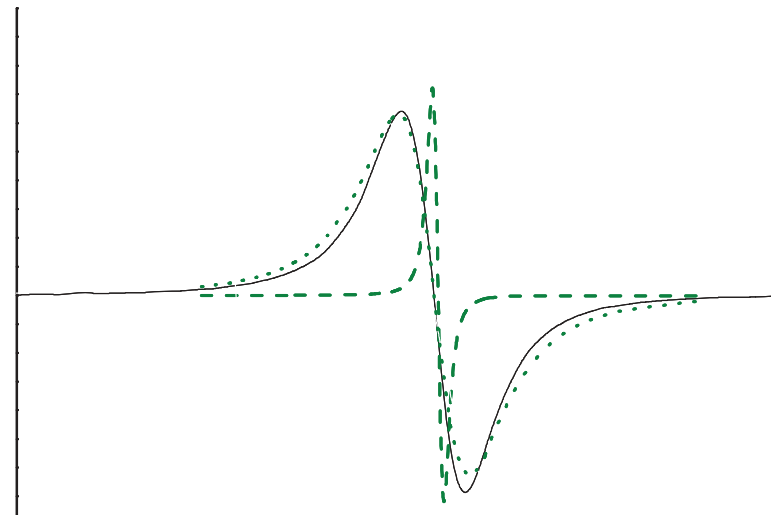

(a)

\begin{tabular}{|l|l|l|l|l|}
\hline 1 & 1 & 1 \\
8.482 & 8.484 & 8.486 & 8.488 & 8.490
\end{tabular}

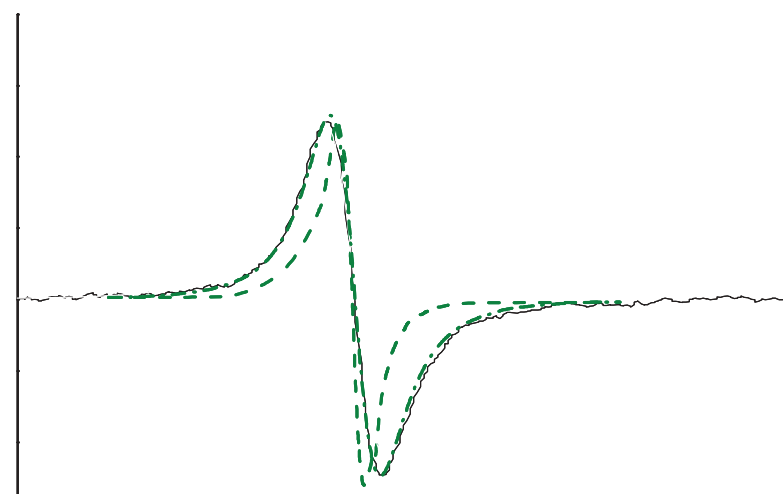

(b)

$\begin{array}{cccc}3.400 & 3.405 & 3.410 & 3.415 \\ & \text { magnetic field } / \mathrm{T} /\end{array}$

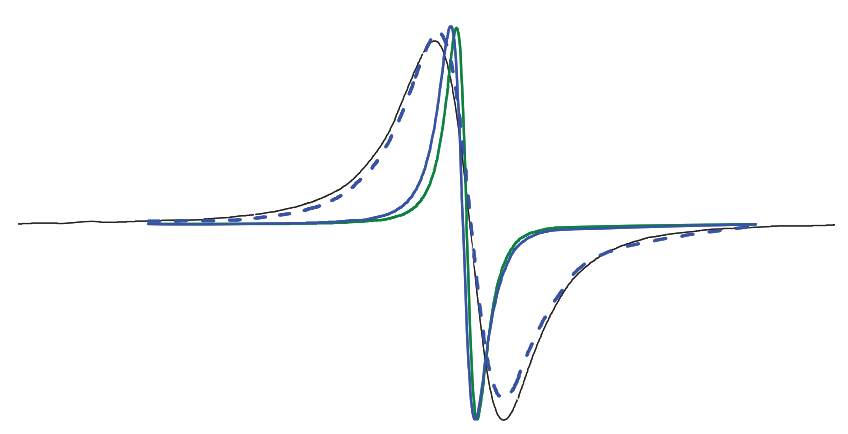

(c)

\begin{tabular}{lllll}
\hline 8.482 & 8.484 & 8.486 & 8.488 & 8.490
\end{tabular}

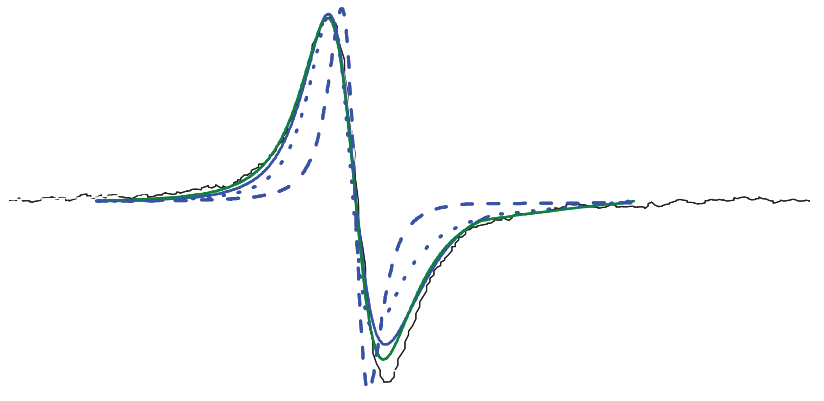

(d)

\begin{tabular}{|c|c|c|c|}
\hline 3.400 & 3.405 & 3.410 & 3.415 \\
\hline
\end{tabular}

FIG. 6. Experimental ESR lineshape for $0.19 \mathrm{mMol}$ P760 at $310 \mathrm{~K}$ (black solid line) for (a, c) $237 \mathrm{GHz}$ and (b, d) $95 \mathrm{GHz}$. (a, b) Theoretical ESR lineshapes for the case $\mathrm{C}$ parameters with $\Delta g=0$ (green dashed line). The green dotted line in panel a corresponds to $\Delta g=0.0025$. In panel (b), the green dashed-dotted line is for $\Delta g=0.009$, (c, d) Theoretical ESR lineshapes for parameters according to case D and $\Delta g=0$ (green line); case $\mathrm{E}$ with $\Delta g=0$ (blue line), $\Delta g=0.0015$ (blue dashed line), $\Delta g=0.007$ (blue dotted line).

do not fit well to the experimental spectra, but the $g$-tensor anisotropy contribution for the moderate $\Delta g=0.0015 \mathrm{im}$ proves considerably the agreement. The ESR spectrum calculated for the higher magnetic field acquires now a proper width [Fig. 7(a)], while the static ZFS features are again reduced [Fig. 7(b)] for the lower magnetic field. In consequence, the high field ESR spectrum becomes somewhat too narrow, but the agreement with experiment is better than in the cases presented in Figs. 4(b) and 6(d). Therefore, the last set of parameters, case F with $\Delta g=0.0015$, leads to the best agreement with the experimental ESR and NMRD data. It should be pointed out at this stage that the quality of this unified analysis presented here is very good indeed compared to similar attempts reported in the literature ${ }^{54,55}$ for larger Gd(III) complexes.

Next, we turn the attention to the second complex of Gd(III), P792. We have performed, in the first step, a 5parameter fit $\left(D_{S}, D_{T}, \tau_{D}, \tau_{M}\right.$, and $\left.r_{I S}\right)$ of the NMRD with fixed $\tau_{R}$ and adjustable $\tau_{M}$. The result is shown in Table I, case $\mathrm{G}$ and in Fig. 8; the fit does not satisfactorily repro- duce the maximum of the relaxation profile. The two complexes, P760 and P792, are structurally similar while the parameters values obtained for the static and transient ZFS for both of them are significantly different. This is not surprising, since the experimental NMRD profiles and ESR spectra show considerable differences as well. However, being aware that the fitting results are not unique, we have attempted to reproduce the NMRD profile not allowing for too large changes in the amplitudes of the static and transient ZFS compared to P760. Such an analysis required allowing for changes in the rotational correlation time, $\tau_{R}$. In Fig. 8, we present also the result of a 5-parameter fit $\left(D_{S}, D_{T}, \tau_{D}, \tau_{R}\right.$, and $r_{I S}$ ) with fixed $\tau_{M}$, case $\mathrm{H}$ in Table I. Now, the static and transient ZFS parameters, $D_{S}$ and $D_{T}$, are comparable with the values obtained for P760 (cases D, E, F), and the distortional correlation time, $\tau_{D}=47.7 \mathrm{ps}$, is similar to the case $\mathrm{F}, \tau_{D}=57.8 \mathrm{ps}$. The obtained $\tau_{R}$ value $(2.9 \mathrm{~ns})$ is now longer. A much better agreement with the experimental data was obtained when allowing for adjustment of both $\tau_{R}$ and $\tau_{M}$. The set of parameters obtained from a 6-parameter fit 


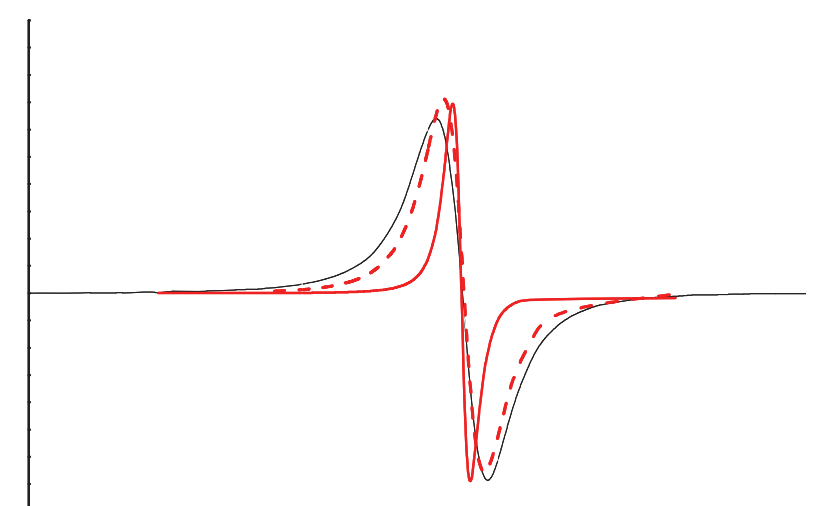

(a)
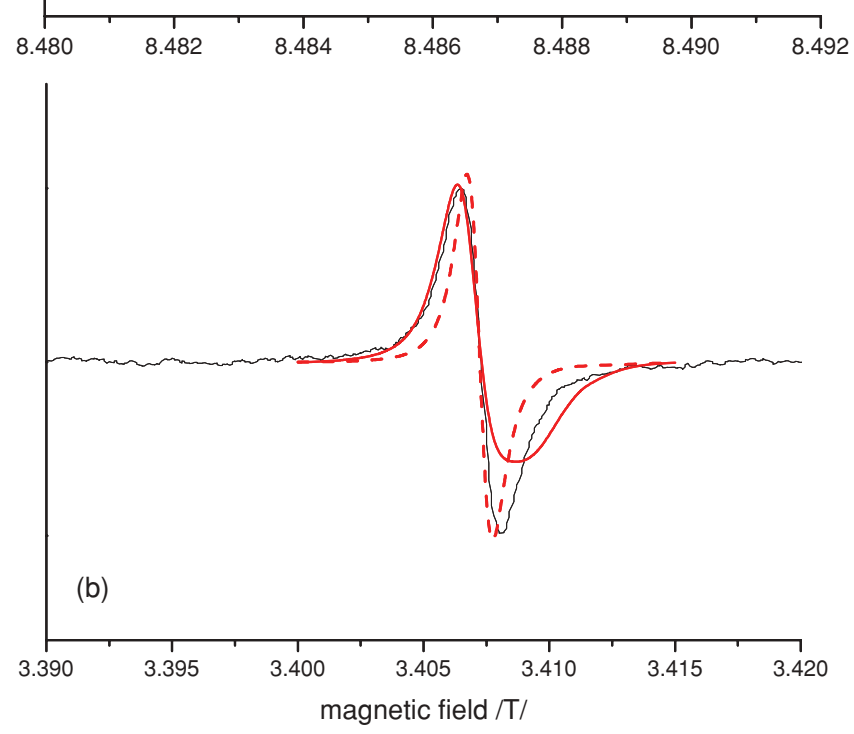

FIG. 7. Experimental ESR lineshape for $0.19 \mathrm{mMol} P 760$ at $310 \mathrm{~K}$ (black solid line) for (a) $237 \mathrm{GHz}$ and (b) $95 \mathrm{GHz}$. Theoretical ESR lineshapes for the case $\mathrm{F}$ parameters and $\Delta g=0$ (red line); $\Delta g=0.0015$ (red dashed line).

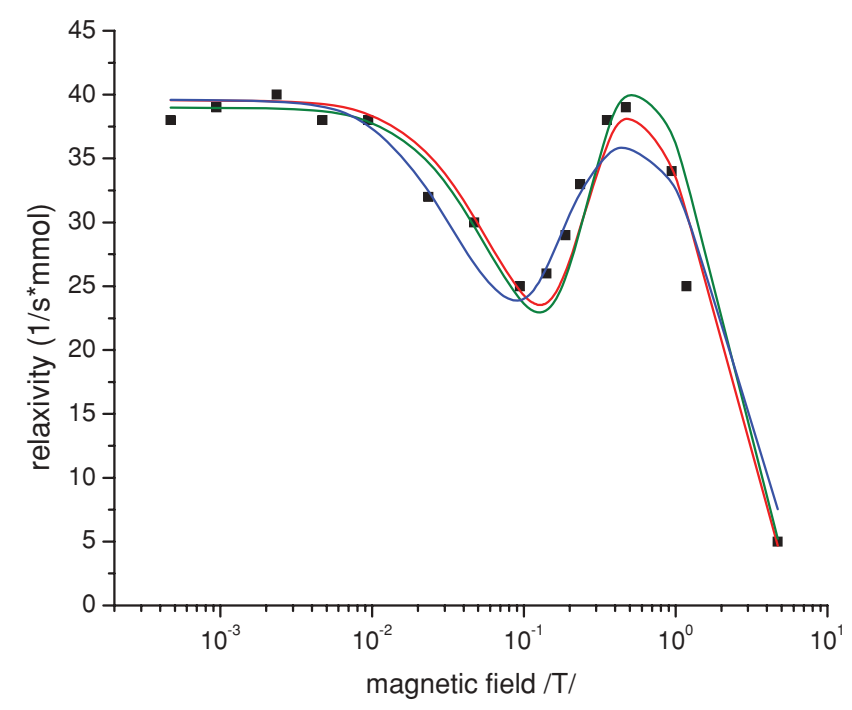

FIG. 8. Experimental NMRD profile for P792 at $310 \mathrm{~K}$ (Ref. 59) (solid squares) and the result of least square fits. Case $\mathrm{G}$, rel.err. $=0.49$ (blue solid line), case H, rel.err. $=0.84$ (green solid line), case I rel.err. $=0.48$ (red solid line).

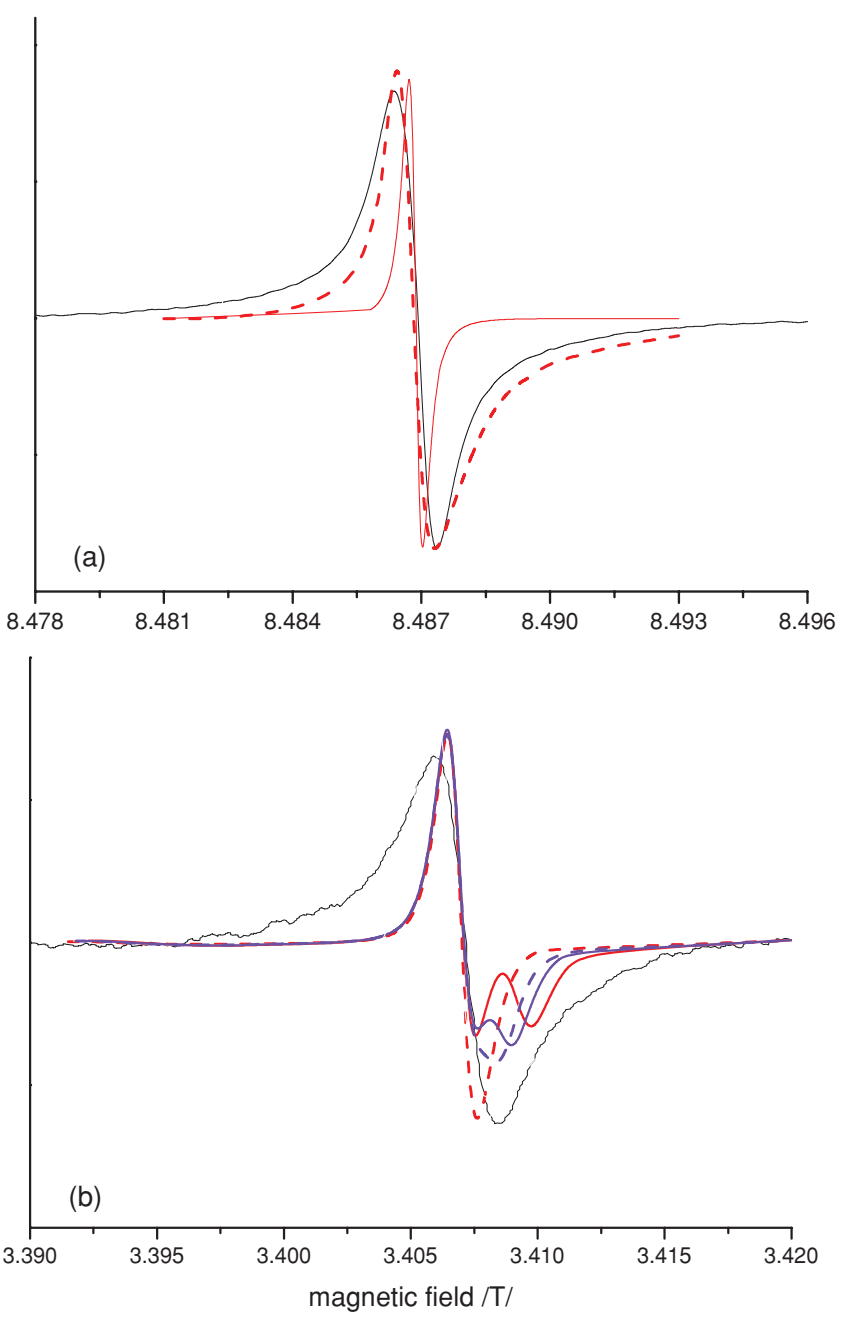

FIG. 9. Experimental ESR lineshape for $0.285 \mathrm{mMol} P 792$ at $310 \mathrm{~K}$ (black solid line) at (a) $237 \mathrm{GHz}$ and (b) $95 \mathrm{GHz}$. Theoretical ESR lineshapes for the case I parameters with $\Delta g=0$ (red solid line), and $\Delta g=0.0013$ (red dashed line). In panel (b), also $\Delta g=0.0005$ (violet solid line), $\Delta g=0.0008$ (purple dashed line).

(with $D_{S}$ and $D_{T}$ kept close to the values for P760), case I in Table I, reproduces the experimental relaxation profile quite well (Fig. 8). The correlation time $\tau_{R}$ gets somewhat longer $\left(\tau_{R}=3.2 \mathrm{~ns}\right)$, but an even larger $\tau_{R}=3.4 \mathrm{~ns}$ was mentioned for P760 when the microviscosity effects were accounted for. ${ }^{60}$ Next, we turn to the ESR spectra corresponding to the case I, Fig. 9. The ESR spectrum calculated for the higher magnetic field excluding the g-anisotropy is, similarly to the cases already considered, too narrow compared to the experimental lineshape. An appropriate contribution of the $g$-tensor anisotropy ( $\Delta g=0.0013$ ) solves the problem relatively well, the corresponding spectrum is also shown in Fig. 9(a). The ESR spectrum calculated for the lower magnetic field $(\Delta g=0)$ shows features created by the static ZFS (the longer rotational correlation time makes them more pronounced) as shown in Fig. 9(b). One can eliminate these effects by introducing the $g$-tensor anisotropy. Illustrative calculations for $\Delta g=0.0005$ and $\Delta g=0.0008$ are shown in Fig. 9(b). The g-tensor anisotropy of $\Delta g=0.0013$ removes them almost completely [Fig. 9(b)] but the calculated ESR line stays too narrow. The static ZFS parameters in line I, 
Table I, can be compared with the values $D, E$ obtained by Benmelouka et al. ${ }^{62}$ from $240 \mathrm{GHz}$ ESR spectra for powder and frozen solution of P792 (mixture of six stereoisomers, in analogy with the material used in the present work) collected at 5 and $4 \mathrm{~K}$, respectively. In the present analysis we do not consider the rhombic term, but we can convert the data of Benmelouka et al. into an effective $D$ value calculated as $D=\sqrt{\frac{3}{2}\left(\frac{2}{3} D_{0}^{2}+2 E_{0}^{2}\right)}=3.53 \times 10^{-2} \mathrm{~cm}^{-1}$, where $D_{0}$ and $E_{0}$ are the frozen solution parameters. This number is close to our $D_{S}$ value of $4.03 \times 10^{-2} \mathrm{~cm}^{-1}$. Benmelouka et al. reported also the strain parameters $\sigma D$ and $\sigma E$, which might be considered related to our $D_{T}$. In view of the approximations involved in the pseudorotation model, we do not believe that a comparison with $\sigma D$ is meaningful.

It is very important in this context to investigate the influence of the concentration of the P792 complex on the ESR lineshape (while the NMRD profiles remain almost unaffected ${ }^{61}$ ). We show ESR spectra for P792 collected for the concentration of $1 \mathrm{mMol}$, and compared with the spectra for $0.285 \mathrm{mMol}$ (the subject of the analysis above), in Fig. 10. One can clearly see that the increased concentration considerably affects the ESR lines at both frequencies, leading in general to line broadening, and that the effect at the higher frequency is larger.
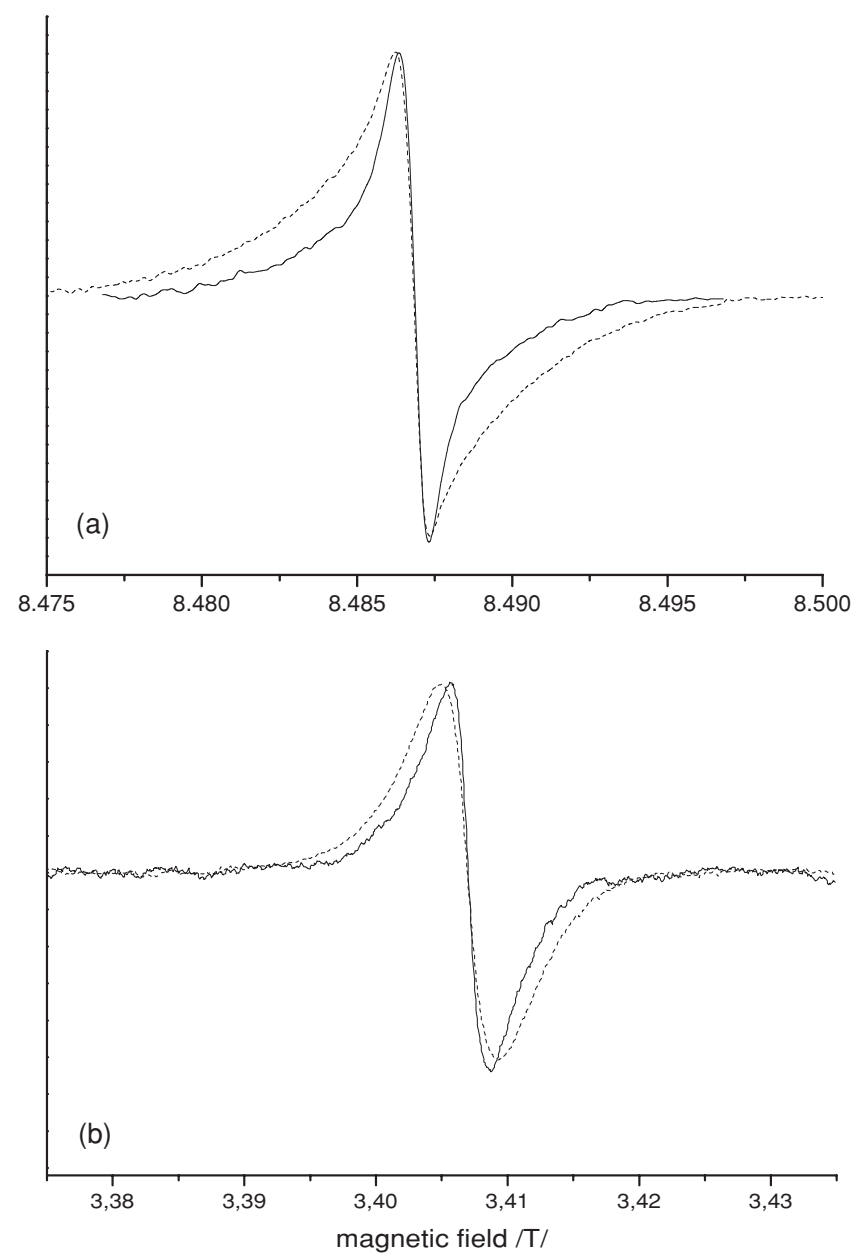

FIG. 10. Experimental ESR lineshapes for P792 at $290 \mathrm{~K}$ for the concentration of $0.285 \mathrm{mMol}$ (solid line) and $1 \mathrm{mMol}$ (dashed line) for the frequency of (a) $237 \mathrm{GHz}$ and (b) $95 \mathrm{GHz}$.
Similar effects were observed in ESR spectra of low-molecular weight $\mathrm{Gd}(\mathrm{III})$ complexes by Powell et al., ${ }^{48}$ who worked, however, at significantly higher concentrations. Our P760 spectra were taken for a somewhat lower concentration than P792. Nevertheless, some influence of interactions between paramagnetic centers of neighboring molecules on the P760 spectra cannot probably be excluded either. It is possible that the concentration effects of this kind can explain the need of assuming somewhat too high g-anisotropies in order to obtain reasonable ESR linewidths at our two fields.

\section{DISCUSSION}

The analysis of ESR spectra and NMRD data presented above has been performed to critically verify the applicability of the pseudorotational model of the transient ZFS. The hierarchy of events in paramagnetic systems (the electron spin dynamics is, in fact, unaffected by the nuclear spins, while the nuclear spin relaxation very significantly depends on the electron spin dynamics) suggests that one should first attempt to analyze ESR spectra, and later use the obtained electron spin parameters to interpret NMRD data resulting from the electron spin dynamics. In our case this approach failed already at the first stage. We were not able to reproduce the ESR spectra at two frequencies within one set of parameters. A contribution of the $g$-tensor anisotropy makes such an analysis possible. Nevertheless, even though the $g$-tensor anisotropy effects are more pronounced for higher magnetic fields it is rather difficult to unambiguously distinguish between the transient ZFS and the $g$-tensor anisotropy contributions to the electron spin dynamics. Therefore, we have chosen to analyze first the NMRD data (especially since the estimates of the exchange lifetime $\tau_{M}$ and the rotational correlation time $\tau_{R}$ were available) and to use the obtained electron spin parameters to interpret the ESR spectra. It was found that the ESR spectra can be treated as a test for the parameters obtained from the NMRD analysis. In some cases the fits of the NMRD were relatively good, while the interpretation of the ESR spectra with these sets of parameters turned out to be unsatisfactory. For other sets of parameters obtained from the NMRD analysis, the interpretation of the ESR spectra was much better, especially when the $g$-tensor anisotropy was included. Since NMRD profiles are determined by many parameters, their analysis is not unambiguous. One cannot expect either that this indirect way of determining the electron spin parameters leads to fully reliable results. On the other hand, the ESR lineshape is very likely influenced by $g$-tensor anisotropy interactions which are much more important for ESR data (especially at high magnetic fields) than for NMRD profiles. A consistent analysis of multifrequency ESR data is therefore a complex issue by itself. Taking into account all the aspects, we have not found a unique strategy for performing a joint analysis of ESR and NMRD data. Nevertheless, when approaching this problem from both sides, we were able to find quite reasonable sets of parameters for both complexes for which a satisfactory agreement for ESR and NMRD was reached. We trust that such an analysis is much more convincing than an interpretation of only the NMRD profiles. 
One point that should be mentioned in this context is our description of rotational motion, which modulates both the DD interaction and the static ZFS. We neglect the internal motions in the case of the DD interactions but allow them in some sense, through the pseudorotational dynamics, for the ZFS. The reason for this difference is that the electron spin dynamics can be expected to be more sensitive to the rapid motions of this kind, since they contribute to spectral densities at high frequencies. For systems with water molecules in the first coordination sphere of a paramagnetic metal ion, this effect of internal dynamics on the DD coupling will hopefully be small and absorbed in the effective nuclear spin-electron spin distance..$^{70,71}$

Given the grossly simplified description of the electron spin dynamics by means of the pseudorotational model, one should in fact not expect an excellent agreement between calculated experimental electron spin and nuclear spin data. The characteristic frequency for the electron spin is three orders of magnitude larger than for the nuclear spin. That means that the nuclear spin senses the wings of the electron spin spectral density. ${ }^{72,73}$ To describe well the wings of a spectral density function, one would expect to need a very accurate model, while the pseudorotational model is highly simplified. In this context the quality of the combined analysis of ESR and NMRD data presented here is, in our opinion, acceptable. The presented examples can be treated as a successful test of the applicability of the pseudorotational model. They also indicate that a further extension of the ESR slow motion theory by including a general form of the g-tensor anisotropy may be relevant. Discussing the effects of g-tensor anisotropy one should be aware of cross-correlations between this interaction and the static ZFS (both interactions are modulated by the rotational motion), effects included naturally in our formulation. One can expect that the symmetry of the g-tensor combined with the relative orientation of the principal frames of both interactions are important for the cross-correlations effects. We consider such an extension of the presented ESR slow motion theory as a possible next step.

We wish also to comment the possible role of spinrotation interaction as an electron spin relaxation mechanism. As mentioned above, this mechanism was included in the study by Powell et al. ${ }^{48}$ Using their simple approach, the estimate of the spin-rotation contribution to the ESR linewidth requires only one additional parameter, an average squared deviation of the principal $g$-tensor element from the free electron value, $\Delta g^{2}$. Taking that $T_{S R}^{-1}=\Delta g^{2} / 9 \tau_{R}$, and setting $\Delta g^{2}=6 \times 10^{-3}\left(\left(2.0015^{2}-4\right)=0.006\right)$, we estimate $T_{S R}^{-1} \cong 3 \times 10^{5} \mathrm{~s}^{-1}$. Our experimental solution ESR spectra have the peak-to-peak linewidth, $\Delta H_{p p}$, of about 0.001-0.0015 T. Using the same relation as Powell et al. ${ }^{48}$ $T_{2 e}^{-1}=g_{L} \mu_{B} \pi \sqrt{3} \Delta H_{p p} / h$ (where $g_{L}=2$ is the Landé $g$-factor and $\mu_{B}$ is the Bohr magneton), we estimate our effective $T_{2 e^{-1}}$ at $1.5 \times 10^{8}$ to $2.3 \times 10^{8} \mathrm{~s}^{-1}$. Compared to these experiment-based values, the estimated spin-rotation contribution to the relaxation rate is indeed negligible.

Finishing this discussion, it is important to stress that the amplitude of the static ZFS combined with the timescale of the molecular tumbling brings the electron spin of the discussed complexes beyond the perturbation limit. Therefore, it was necessary to apply the general slow motion theory to interpret the NMRD as well as the ESR data. Any kind of perturbation treatment would very likely lead to larger discrepancies between the experimental data and the results of such a treatment might be misinterpreted as a failure of the pseudorotational model. Seen in that perspective, the present contribution is an important step towards understanding solution ESR lineshapes for high-spin transition metal ion and lanthanide complexes.

\section{CONCLUDING REMARKS}

Aiming at a verification of the pseudorotational model of the transient ZFS interaction, we have adopted the slow motion theory (applied so far to NMRD relaxation profiles) to the analysis of ESR spectral lineshapes. In this way, we have developed a general tool for interpreting ESR and NMRD data for paramagnetic systems for a large variety of interaction strengths and motional conditions (including the slow motion theory of the outer-sphere $\left.\mathrm{PRE}^{38,39}\right)$. The theory was used to interpret in a consistent way (within one set of parameters) NMRD profiles and ESR spectra at 95 and $237 \mathrm{GHz}$ for two Gd(III) complexes, denoted as P760 and P792. The analysis indicated an additional mechanism contributing to the electron spin dynamics which was attributed to the $g$-tensor anisotropy. Thus, the slow motion theory of ESR spectra has been extended by including g-tensor anisotropy effects (under the simplifying assumption that $g_{x x}=g_{y y}$ and $g_{\text {eff }}=2$ ). The unified interpretation of the ESR and NMRD led to an acceptable agreement with the experimental data, supporting the concept of the pseudorotational model as capturing the essential features of the electron spin dynamics.

\section{ACKNOWLEDGMENTS}

This work was financed by Polish Ministry of Science and Education, grant No. N N202 105936, and by the Swedish Science Research Council. The project was also supported by Grant Number 5P41RR016292 (at Cornell) from the National Center for Research Resources (NCRR), a component of the National Institutes of Health (NIH). We are indebted to Guerbet (Roissy, France) for the gift of the P760 and P792 samples for ESR spectroscopy and to Professor Robert Muller and Professor Nikolas Benetis for valuable discussions.

\section{APPENDIX}

Under the assumption that $g_{x x}=g_{y y}, \quad g_{\text {eff }}$ $=\frac{1}{3}\left(g_{x x}+g_{y y}+g_{z z}\right)=2 \quad\left(g_{x x}, g_{y y}, g_{z z}\right.$ are cartesian components of the $g$-tensor) and that the principal axis system of the $g$-tensor anisotropy coincides with the $\left(P_{S}\right)$ frame, the electron spin Zeeman interaction in the laboratory frame takes the form:

$$
\begin{aligned}
H_{Z}(S)= & \omega_{S} S_{z}+H_{Z}^{\Delta g}(S)=\omega_{S} S_{z}+\omega_{S} \frac{\Delta g}{g_{\text {eff }}}\left[2 D_{0,0}^{2}\left(\Omega_{P_{S} L}\right) S_{z}\right. \\
& \left.-\sqrt{\frac{3}{2}} D_{0,1}^{2}\left(\Omega_{P_{S} L}\right) S_{-}+\sqrt{\frac{3}{2}} D_{0,-1}^{2}\left(\Omega_{P_{S} L}\right) S_{+}\right]
\end{aligned}
$$


with $\Delta g=g_{\text {eff }}-g_{z z}$. To obtain the matrix representation of the Liouville operator $\hat{L}_{Z}^{\Delta g}(S)$, associated with the Hamiltonian $H_{Z}^{\Delta g}(S)$, in the basis $\left.\left.\left\{\mid O_{i}\right)\right\}=\{\mid A B C) \otimes \mid L K M\right) \otimes$ $\mid \Sigma \sigma)\}$ one applies the Wigner-Eckart theorem. ${ }^{74}$ This leads to the following expression for the matrix elements:

$$
\begin{aligned}
\left(O_{i}\left|\hat{\hat{L}}_{Z}^{\Delta g}\right| O_{j}\right) & =\left(A^{\prime} B^{\prime} C^{\prime}\left|\left(L^{\prime} K^{\prime} M^{\prime}\left|\left(\Sigma^{\prime} \sigma^{\prime}\left|\hat{\hat{L}}_{Z}^{\Delta g}\right| A B C\right)\right| L K M\right)\right| \Sigma \sigma\right) \\
& =\delta_{A A^{\prime}} \delta_{B B^{\prime}} \delta_{C C^{\prime}} \omega_{S} \frac{\Delta g}{g_{\mathrm{eff}}}(-1)^{\sigma^{\prime}+K^{\prime}-M^{\prime}}\left[(-1)^{\Sigma^{\prime}+\Sigma+1}-1\right] \\
& \times \sqrt{(2 S+1)(S+1) S\left(2 L^{\prime}+1\right)(2 L+1)\left(2 \Sigma^{\prime}+1\right)(2 \Sigma+1)}\left(\begin{array}{ccc}
L^{\prime} & 1 & L \\
-K^{\prime} & 0 & K
\end{array}\right)\left\{\begin{array}{ccc}
\Sigma^{\prime} & 1 & \Sigma \\
S^{\prime} & S & S
\end{array}\right\} \\
& \times\left\{2\left(\begin{array}{ccc}
\Sigma^{\prime} & 1 & \Sigma \\
-\sigma^{\prime} & 0 & \sigma
\end{array}\right)\left(\begin{array}{ccc}
L^{\prime} & 1 & L \\
-K^{\prime} & 0 & K
\end{array}\right)+\frac{3}{\sqrt{2}}\left[\left(\begin{array}{ccc}
\Sigma^{\prime} & 1 & \Sigma \\
-\sigma^{\prime} & 1 & \sigma
\end{array}\right)\right.\right. \\
& \left.\left.\times\left(\begin{array}{ccc}
L^{\prime} & 1 & L \\
-K^{\prime} & 1 & K
\end{array}\right)+\left(\begin{array}{ccc}
\Sigma^{\prime} & 1 & \Sigma \\
-\sigma^{\prime} & -1 & \sigma
\end{array}\right)\left(\begin{array}{ccc}
L^{\prime} & 1 & L \\
-K^{\prime} & -1 & K
\end{array}\right)\right]\right\}
\end{aligned}
$$

where () and \{\} denote 3-j and 6-j symbols, ${ }^{74}$ respectively.

In the numerical implementation of the NMRD slow motion theory, a considerable number of the $\mid A B C) \otimes \mid L K M) \otimes \mid \Sigma \sigma)$ states could be excluded due to symmetry properties of the $3-\mathrm{j}$ and $6-\mathrm{j}$ symbols which considerably reduces the size of the $\left[M_{\mathrm{NMRD}}\right]$ matrix. For example, the vector $\mid 000) \otimes \mid 000) \otimes \mid 1-1$ ) associated with ESR spectrum is not explicitly represented in this matrix. Now, to calculate ESR spectra, some of the selection rules need to be removed and that leads to an increase of the size of the $\left[M_{\mathrm{ESR}}\right]$ matrix compared to $\left[M_{\mathrm{NMRD}}\right]$. The $g$-tensor anisotropy causes a further enlargement of the $\left[M_{\mathrm{ESR}}\right]$ matrix size, since more basis vectors have to be explicitly accounted for.

${ }^{1}$ N. Bloembergen and L. O. Morgan, J. Chem. Phys. 34, 842 (1961).

${ }^{2}$ I. Bertini, C. Luchinat, and G. Parigi, Solution NMR of Paramagnetic Molecules (Elsevier, Amsterdam, 2001).

${ }^{3}$ D. Kruk, Theory of Evolution and Relaxation of Multi-Spin Systems (Arima, Bury St Edmunds, 2007).

${ }^{4}$ J. Kowalewski, D. Kruk, and G. Parigi, Adv. Inorg. Chem. 57, 41 (2005).

${ }^{5}$ C. P. Slichter, Principles of Magnetic Resonance (Springer-Verlag, Berlin, 1990).

${ }^{6}$ M. Rubinstein, A. Baram, and Z. Luz, Mol. Phys. 20, 67 (1971).

${ }^{7}$ P.-O. Westlund, Mol. Phys. 85, 1165 (1995).

${ }^{8}$ E. Strandberg and P.-O. Westlund, J. Magn. Reson. A 122, 179 (1996).

${ }^{9}$ A. G. Redfield, Adv. Magn. Reson. 1, 1 (1965).

${ }^{10}$ A. G. Redfield, in Encyclopedia of Nuclear Magnetic Resonance (edited by D. M. Grant and R. K. Harris (Wiley, Chichester, 1996), 4085.

${ }^{11}$ I. Bertini, J. Kowalewski, C. Luchinat, T. Nilsson, and G. Parigi, J. Chem. Phys. 111, 5795 (1999).

${ }^{12}$ D. Kruk, T. Nilsson, and J. Kowalewski, Phys. Chem. Chem. Phys. 3, 4907 (2001).

${ }^{13}$ T. Bayburt and R. R. Sharp, J. Chem. Phys. 92, 5892 (1990).

${ }^{14}$ R. R. Sharp, J. Chem. Phys. 93, 6921 (1990).

${ }^{15}$ R. R. Sharp, J. Magn. Reson. 100, 491 (1992).

${ }^{16}$ R. R. Sharp, J. Chem. Phys. 98, 912 (1993).
${ }^{17}$ R. R. Sharp, J. Chem. Phys. 98, 2507 (1993).

${ }^{18}$ R. R. Sharp, J. Chem. Phys. 98, 6092 (1993).

${ }^{19}$ S. Rast, A. Borel, L. Helm, E. Belorizky, P. H. Fries, and E. A. Merbach, J. Am. Chem. Soc. 123, 2637 (2001).

${ }^{20}$ S. Rast, P. H. Fries, and J. Belorizky, J. Chem. Phys. 113, 8724 (2000).

${ }^{21}$ S. Rast, P. H. Fries, E. Belorizky, A. Borel, L. Helm, and E. A. Merbach, J. Chem. Phys. 115, 7554 (2001).

${ }^{22}$ A. Borel, F. Yerly, L. Helm, and E. A. Merbach, J. Am. Chem. Soc. 124, 2042 (2002).

${ }^{23}$ J. H. Freed, in Spin Labeling: Theory and Applications Edited by L. J. Berliner (Academic, N.Y. 1976), p. 53.

${ }^{24}$ G. Moro and J. H. Freed J. Chem. Phys. 74, 3757 (1981).

${ }^{25}$ D. J. Schneider and J. H. Freed, Adv. Chem. Phys. 73, 387 (1989).

${ }^{26}$ D. J. Schneider and J. H. Freed, Biol. Magn. Reson. 8, 1 (1989).

${ }^{27}$ Z. C. Liang and J. H. Freed, J. Phys. Chem. B 103, 6384 (1999).

${ }^{28}$ A. A. Nevzorov and J. H. Freed, J. Chem. Phys. 112, 1413 (2000).

${ }^{29}$ N. Benetis, J. Kowalewski, L. Nordenskiöld, H. Wennerström, and P.-O. Westlund, Mol. Phys. 48, 329 (1983).

${ }^{30}$ T. Nilsson and J. Kowalewski, J. Magn. Reson. 146, 345 (2000).

${ }^{31}$ K. Åman and P.-O. Westlund, Mol. Phys. 102, 1085 (2004).

${ }^{32}$ K. Åman and P.-O. Westlund, Phys. Chem. Chem. Phys. 9, 691 (2007).

${ }^{33}$ E. Belorizky, P. H. Fries, L. Helm, J. Kowalewski, D. Kruk, R. R. Sharp, and P.-O. Westlund, J. Chem. Phys. 128, 052315 (2008).

${ }^{34}$ P. H. Fries and E. Belorizky, J. Chem. Phys. 126, 204503 (2007).

${ }^{35}$ S. M. Abernathy and R. R. Sharp, J. Chem. Phys. 106, 9032 (1997).

${ }^{36}$ N. Schaefle and R. Sharp, J. Chem. Phys. 121, 5387 (2004).

${ }^{37}$ N. Schaefle and R. Sharp, J. Magn. Reson. 176, 160 (2005).

${ }^{38}$ D. Kruk and J. Kowalewski, J. Chem. Phys. 130, 174104 (2009).

${ }^{39}$ L. P. Hwang and J. H. Freed, J. Chem. Phys. 63, 4017 (1975).

${ }^{40}$ Y. Ayant, E. Belorizky, J. Alizon, and J. Gallice, J. Phys. (Paris) 36, 991 (1975).

${ }^{41}$ J. H. Freed, G. V. Bruno and, C. Polnaszek, J. Chem. Phys. 55, 5270 (1971).

${ }^{42}$ P.-O. Westlund and H. Wennerström, Phys. Chem. Chem. Phys. 12, 201 (2010).

${ }^{43}$ M. Odelius, C. Ribbing, and J. Kowalewski, J. Chem. Phys. 103, 1800 (1995).

${ }^{44}$ M. Odelius, C. Ribbing, and J. Kowalewski, J. Chem. Phys. 104, 3181 (1996).

${ }^{45}$ M. Lindgren, A. Laaksonen, and P.-O. Westlund, Phys. Chem. Chem. Phys. 11, 10368 (2009).

${ }^{46}$ D. Kruk and J. Kowalewski, J. Chem. Phys. 116, 4079 (2002). 
${ }^{47}$ D. Kruk, J. Kowalewski, and P.-O. Westlund, J. Chem. Phys. 121, 2215 (2004).

${ }^{48}$ D. H. Powell, O. M. Ni Dhubhghaill, D. Pubanz, L. Helm, Y. S. Lebedev, W. Schlaepfer, and A. E. Merbach, J. Am. Chem. Soc. 118, 9333 (1996).

${ }^{49}$ E. Toth, F. Connac, L. Helm, K. Adzamli, and A. E. Merbach, Eur. J. Inorg. Chem., 2017 (1998).

${ }^{50}$ E. Zitha-Bovens, R. M. Muller, S. Laurent, L. Vander Elst, C. F .G. C. Geraldes, H. van Bekkum, and J. A. Peters, Helv. Chim. Acta 88, 618 (2005).

${ }^{51}$ X. Z. Zhou and P.-O. Westlund, Spectrochim. Acta A62, 76 (2005).

${ }^{52}$ X. Z. Zhou and P.-O. Westlund, Spectrochim. Acta A62, 335 (2005).

${ }^{53}$ F. A. Dunand, A. Borel, and L. Helm, Inorg. Chem. Commun. 5, 811 (2002).

${ }^{54}$ X. Z. Zhou and P.-O. Westlund, J. Magn. Reson. 173, 75 (2005).

${ }^{55}$ X. Z. Zhou, P. Caravan, R. B. Clarkson, and P.-O. Westlund, J. Magn. Reson. 167, 147 (2004).

${ }^{56}$ M. Benmelouka, J. Van Tol, A. Borel, M. Port, L. Helm, L. C. Brunel, and A. E. Merbach, J. Am. Chem. Soc. 128, 7807 (2006).

${ }^{57}$ A. Borel, R. B. Clarkson, and R. L. Belford, J. Chem. Phys. 126, 054510 (2007).

${ }^{58}$ R. B. Clarkson, A. I. Smirnov, T. I. Smirnova, H. Kang, R. L. Belford, K. Earle, and J. H. Freed, Mol. Phys. 95, 1325 (1998).

${ }^{59}$ A. Borel, H. Kang, C. Gateau, M. Mazzanti, R. B. Clarkson, and R. L. Belford, J. Phys. Chem. A 110, 12434 (2006).

${ }^{60}$ L. Vander Elst, M. Port, I. Raynal, C. Simonot, and R. N. Muller, Eur. J. Inorg. Chem. 2003, 2495.
${ }^{61}$ L. Vander Elst, I. Raynal, M. Port, P. Tisnes, and R. N. Muller, Eur. J. Inorg. Chem. 2005, 1142.

${ }^{62}$ M. Benmelouka, J. Van Tol, A. Borel, S. Nellutla, M. Port, L. Helm, L.-C. Brunel, and A. E. Merbach, Helv. Chim. Acta 92, 2173 (2009).

${ }^{63}$ J. Kowalewski, A. Egorov, D. Kruk, A. Laaksonen, S. Nikkhou Aski, G. Parigi, and P.-O. Westlund, J. Magn. Reson. 195, 103 (2008).

${ }^{64}$ S. K. Misra, S. I. Andronenko, and T. Yu. Chemekova, Phys. Rev. B 67, 214411 (2003).

${ }^{65}$ A. Borel, S. Laus, A. Ozarowski, C. Gateau, A. Nonat, M. Mazzanti, and L. Helm, J. Phys. Chem. A 111, 5399 (2007).

${ }^{66}$ K. V. Vasavada and B. D. Nageswara Rao, J. Magn. Reson. 81, 275 (1989).

${ }^{67}$ I. Bertini, C. Luchinat, and K. V. Vasavada, J. Magn. Reson. 89, 243 (1990).

${ }^{68}$ T. Nilsson, J. Svoboda, P.-O. Westlund, and J. Kowalewski, J. Chem. Phys. 109, 6364 (1998).

${ }^{69}$ T. Nilsson and J. Kowalewski, Mol. Phys. 98, 1617 (2000); Erratum, ibid 99, 369 (2001)

${ }^{70}$ E. R. Henry and A. Szabo, J. Chem. Phys. 82, 4753 (1985).

${ }^{71}$ J. Kowalewski, M. Effemey, and J. Jokisaari, J. Magn. Reson. 157, 171 (2002).

${ }^{72}$ S. H. Koenig, J. Magn. Reson. 31, 1 (1978).

${ }^{73}$ S. H. Koenig, J. Magn. Reson. 47, 441 (1982).

${ }^{74}$ A. R. Edmonds, Angular Momentum in Quantum Mechanics (Princeton University, Princeton, 1974) 